\title{
Low-Dimensional Approximations of High-Dimensional Asset Price Models*
}

\author{
Martin Redmann ${ }^{\dagger}$, Christian Bayer ${ }^{\ddagger}$, and Pawan Goyal ${ }^{\S}$
}

\begin{abstract}
We consider high-dimensional asset price models that are reduced in their dimension in order to reduce the complexity of the problem or the effect of the curse of dimensionality in the context of option pricing. We apply model order reduction (MOR) to obtain a reduced system. MOR has been previously studied for asymptotically stable controlled stochastic systems with zero initial conditions. However, stochastic differential equations modeling price processes are uncontrolled, have nonzero initial states and are often unstable. Therefore, we extend MOR schemes and combine ideas of techniques known for deterministic systems. This leads to a method providing a good pathwise approximation. After explaining the reduction procedure, the error of the approximation is analyzed and the performance of the algorithm is shown conducting several numerical experiments. Within the numerics section, the benefit of the algorithm in the context of option pricing is pointed out.
\end{abstract}

Key words. model order reduction, Black Scholes model, Heston model, option pricing

AMS subject classifications. Primary, 91G20, 91G60, 93A15; Secondary, 60H10, 65C30

DOI. $10.1137 / 20 \mathrm{M} 1325666$

1. Introduction. In finance we often encounter high-dimensional models, since the underlying markets are usually high-dimensional. For instance, in equity, take all stocks comprising the S \& P 500 index (SPX). Fixed income markets exhibit a myriad of different relevant interest rates. All these are, obviously, only small snapshots of even larger markets. Of course, in many situations, we are only interested in a tiny fraction of these markets, which can be adequately modeled by a low-dimensional stochastic process. Moreover, if we are interested in derivatives on SPX, for example, then we may just model the index itself, disregarding the fine structure. On the other hand, if we consider a larger portfolio, this may not be possible without introducing inconsistencies in the model.

From a numerical perspective, high-dimensional models pose severe difficulties. Indeed, many traditional computational tools suffer from the curse of dimensionality, which essentially states that the computational work required to compute the relevant quantity of interest up to a prescribed error tolerance grows exponentially in the dimension $n$ of the model. Most methods for discretizing partial differential equations (such as finite element and finite difference methods) suffer from the curse of dimensionality, as do Fourier based methods.

\footnotetext{
* Received by the editors March 16, 2020; accepted for publication (in revised form) September 22, 2020; published electronically January 5, 2021.

https://doi.org/10.1137/20M1325666

${ }^{\dagger}$ Institute of Mathematics, Martin Luther University Halle-Wittenberg, Theodor-Lieser-Str. 5, 06120 Halle (Saale), Germany (martin.redmann@mathematik.uni-halle.de).

${ }^{\ddagger}$ Weierstraß Institute for Applied Analysis and Stochastics, Mohrenstr. 39, 10117 Berlin, Germany (christian. bayer@wias-berlin.de).

${ }^{\S}$ Max Planck Institute for Dynamics of Complex Technical Systems, Sandtorstr. 1, 39106 Magdeburg, Germany (goyalp@mpi-magdeburg.mpg.de).
} 
Even many deterministic sampling methods (i.e., tree methods, quasi Monte Carlo) suffer from the curse of dimensionality in one way or another. ${ }^{1}$ The notable exception is, of course, Monte Carlo simulation.

One way to overcome the numerical burden in high-dimensional models is Model order reduction (MOR) $[1,2,12]$. MOR is a technique in numerical analysis in order to construct low-dimensional surrogate models that allow to approximate the quantity of interest with the desired accuracy. As MOR takes the specific quantity of interest into account, reduced models for different options will generally be different. (The specific method introduced later will, however, not depend, e.g., on the specific strike price.)

To fix ideas, suppose that we are given an $n$ dimensional stochastic volatility model with asset price processes $S(t) \in \mathbb{R}^{n}$ and the corresponding stochastic variance processes $v$-which will be one-dimensional in our numerical examples. Consider an option with payoff $g(C S)$, where $C \in \mathbb{R}^{p \times n}$ and $g: \mathbb{R}^{p} \rightarrow \mathbb{R}$ possibly nonlinear, where we assume that $p \ll n$. For instance, we have $p=1$ for basket options. Our goal is to construct a Markov process $\tilde{x}$ taking values in $\mathbb{R}^{\tilde{n}}$ - with $\tilde{n} \ll n$ - and a matrix $C_{1} \in \mathbb{R}^{p \times \tilde{n}}$ such that the processes $C S$ and $C_{1} \tilde{x}$ are close in $L^{2}$. This usually ensures a good approximation of the payoff, i.e., $g(C S) \approx g\left(C_{1} \tilde{x}\right)$. In this paper, we present a general strategy for identifying such processes $\tilde{x}$. We also provide numerical evidence of successful MOR in several financial applications, in the sense that relative errors of the order of $10^{-4}$ are regularly achieved with very small $\tilde{n}$ even when $n \geq 100$. It should be noted here that we only propose a MOR technique for the asset process $S$ in this paper, but not for the variance process $v$. This is due to the generally nonlinear dynamics of the variance process, which would require more complicated MOR strategies and will be explored in future work.

Before explaining the MOR strategy in detail, some conclusions can already be made based on the fundamental idea. First note that MOR should not be confused with Markovian projection; see $[14,25]$. The underlying problem is, of course, that the process $C S$ itself is a natural candidate for a reduced model, but it generally lacks the Markov property. There is, however, a Markov process $\hat{x}$ taking values in $\mathbb{R}^{p}$ such that $C S(t)$ and $\hat{x}(t)$ have the same distribution for every $t$. This means that European option prices based on $\hat{x}$ correspond exactly to the prices in the full model. The coefficients of $\hat{x}$ are, however, not trivial to obtain. Nonetheless, there has been continuous interest in the financial community in applications of Markovian projections; see, for instance, [26] and [4] for two recent examples.

In contrast, the surrogate model $\tilde{x}$ is often easier to construct than the Markovian projection $\hat{x}$. Moreover, our construction provides that $\tilde{x}$ is close to $C S$ on path-space, which directly allows the application to American option pricing. This comes at the price of being only an approximation, though. Moreover, MOR may provide good low dimensional surrogate models even in situations when there is no natural low-dimensional intermediate process, i.e., when $p \approx n$ as of above.

Remark 1. Generally, the surrogate model $\tilde{x}$ does not have any specific financial interpretation. Hence, its only justification is the approximation quality with respect to the quantity of interest.

\footnotetext{
${ }^{1}$ It is worth pointing out that the effective dimension for Quasi Monte Carlo and Monte Carlo method is, in fact, $n$ multiplied by the number of time-steps, if time discretization is needed.
} 
Additionally, there are many dimension reduction techniques in the computational finance literature working at the level of a numerical approximation rather than the model itself, i.e., in contrast to MOR or Markovian projections, no lower-dimensional model is ever considered, but rather the dimensions of certain numerical approximations are reduced. A good, clarifying example might be the use of sparse grid approximations for numerical integration or solving partial differential equations. The underlying observation is, of course, that a one-dimensional grid of size $N$ is turned into a "tensor-product" grid of size $N^{n}$ in dimension $n$. This explosion can often be avoided by a careful choice of a sparse "subgrid." Indeed, under suitable (often quite demanding) regularity conditions, similar accuracy can be achieved with sparse grids of size asymptotically proportional to $N \log (N)^{n}$. We refer to [16] for a general exposition of sparse grid methods, in particular for solving PDEs, and to [5, 23] for applications of sparse grid quadrature methods in finance. If sparse grids are used to discretize pricing PDEs, for example, then the dimensions of the resulting system of linear equations is drastically reduced as compared to the tensor product grid by using available low-dimensional structures in the discretized equation. In contrast, MOR identifies lower-dimensional effective structures already in the continuous model, before any discretization, allowing us to use less sophisticated numerical methods in a low-dimensional surrogate models, in which all components are actually important.

A somewhat complementary method for solving high-dimensional problems in finance is based on machine learning, in particular deep neural networks. These methods are praised for their ability to handle very high-dimensional problems, seemingly breaking the curse of dimensionality. Hence, they often offer effective alternative computational approaches, even without explicit dimension reduction. We refer to $[15,27]$ for two recent examples of applications in computational finance.

Outline of the paper. After setting the stage in section 2, we introduce techniques to provide quantitative estimates of "importance" of projections of the state for the dynamics of the process in section 3. These quantitative estimates are then used in section 4 to identify especially efficient reduced models. We continue to provide error bounds in section 5 . Numerical experiments are reported in section 6 , followed by concluding remarks in section 7 . Some general definitions and technical proofs are presented in an appendix.

2. Setting and covariance functions. Let $W=\left(W_{1}, \ldots, W_{q}\right)^{T}$ be an $\mathbb{R}^{q}$-valued with mean zero Wiener process with covariance matrix $\mathbf{K}=\left(k_{i j}\right)$, i.e., $\mathbb{E}\left[W(t) W^{T}(t)\right]=\mathbf{K} t$ for $t \in[0, T]$, where $T>0$ is the terminal time. Suppose that $W$ and all stochastic process appearing in this paper are defined on a filtered probability space $\left(\Omega, \mathcal{F},\left(\mathcal{F}_{t}\right)_{t \in[0, T]}, \mathbb{P}\right) .{ }^{2}$ In addition, we assume $W$ to be $\left(\mathcal{F}_{t}\right)_{t \in[0, T]}$-adapted and the increments $W(t+h)-W(t)$ to be independent of $\mathcal{F}_{t}$ for $t, h \geq 0$. We consider the following large-scale Heston type model:

$$
\begin{aligned}
d x(t) & =A x(t) d t+\sum_{i=1}^{q} \sqrt{v(t)} N_{i} x(t) d W_{i}(t), \quad x(0)=x_{0}=B z, \\
y(t) & =C x(t), \quad t \in[0, T]
\end{aligned}
$$

\footnotetext{
${ }^{2}\left(\mathcal{F}_{t}\right)_{t \in[0, T]}$ shall be right continuous and complete.
} 
where $A, N_{i} \in \mathbb{R}^{n \times n}$ and $C \in \mathbb{R}^{p \times n}$. Moreover, the set of initial conditions, in which we are interested, is spanned by the columns of a matrix $B \in \mathbb{R}^{n \times m}$; i.e., there is a vector $z \in \mathbb{R}^{m}$ such that $x_{0}=B z$. This assumption allows to construct a reduced-order system that performs well for several initial states. However, there are many financial applications where only a single $x_{0}$ is of interest. Then, we have $B=x_{0}$ and $z=1$. The scalar $\left(\mathcal{F}_{t}\right)_{t \in[0, T]}$-adapted stochastic process $(v(t))_{t \in[0, T]}$ is nonnegative, $\mathbb{P}$-almost surely (a.s.) bounded from above by a constant $c>0$ and called variance process. The variance process is assumed to be bounded for theoretical considerations below. Practically, boundedness is less relevant. The state dimension $n$ is assumed to be large and the quantity of interest $y$ is rather low-dimensional; i.e., $p \ll n$.

Below, the dependence of the state variable on $x_{0}$ is sometimes indicated by writing $x\left(t ; x_{0}\right), t \in[0, T]$, for the solution to (2.1a). Furthermore, we write $M_{1} \leq M_{2}$ for two symmetric matrices $M_{1}$ and $M_{2}$ if $M_{2}-M_{1}$ is symmetric positive semidefinite. In order to identity the important states in system (2.1), the covariance function and an upper bound for the covariance will be of interest. Therefore, we formulate the following lemmas.

Lemma 2.1. The matrix-valued function $\mathbb{E}\left[x\left(t ; x_{0}\right) x^{T}\left(t ; x_{0}\right)\right], t \in[0, T]$, is a solution to

$$
\dot{X}(t) \leq A X(t)+X(t) A^{T}+c \sum_{i, j=1}^{q} N_{i} X(t) N_{j}^{T} k_{i j}, \quad X(0)=x_{0} x_{0}^{T},
$$

where $k_{i j}$ is the ijth entry of the covariance matrix $\mathbf{K}$.

Proof. The proof is given in Appendix B.1.

We denote the solution to (2.1a) by $x_{c}$ if the process $v$ is replaced by its upper bound $c$ $(v \equiv c)$. We call $(2.1)$ Black Scholes model in case the volatility is constant. The covariance function of $x_{c}$ can be derived through the identity given in the following lemma.

Lemma 2.2. The matrix-valued function $\mathbb{E}\left[x_{c}\left(t ; x_{0}\right) x_{c}^{T}\left(t ; x_{0}\right)\right], t \in[0, T]$, satisfies

$$
\dot{X}_{c}(t)=A X_{c}(t)+X_{c}(t) A^{T}+c \sum_{i, j=1}^{q} N_{i} X_{c}(t) N_{j}^{T} k_{i j}, \quad X_{c}(0)=x_{0} x_{0}^{T} .
$$

Proof. The statement of this lemma is a special case of [35, Lemma 2.1].

We now formulate a Gronwall type lemma for matrix differential inequalities involving resolvent positive operators. We refer to Appendix A for a definition of these operators.

Lemma 2.3. Suppose that $L$ is a resolvent positive operator on the space of symmetric matrices. Let the matrix-valued function $X(t) \geq 0, t \in[0, T]$, satisfy

$$
\dot{X}(t) \leq L(X(t))
$$

and let $Z(t) \geq 0, t \in[0, T]$, be the solution to the matrix differential equation

$$
\dot{Z}(t)=L(Z(t)) .
$$

If $X(0) \leq Z(0)$, we have that $X(t) \leq Z(t)$ for all $t \in[0, T]$. 
Proof. The proof of this theorem for a special resolvent positive operator is given in [36, Lemma 3.3]. In order to render this paper as self-contained as possible, the proof is stated in Appendix B.2 using the same arguments.

Lemma 2.3 together with Lemmas 2.1 and 2.2 implies that

$$
\mathbb{E}\left[x\left(t ; x_{0}\right) x^{T}\left(t ; x_{0}\right)\right] \leq \mathbb{E}\left[x_{c}\left(t ; x_{0}\right) x_{c}^{T}\left(t ; x_{0}\right)\right],
$$

since $L(X):=A X+X A^{T}+c \sum_{i, j=1}^{q} N_{i} X N_{j}^{T} k_{i j}$ defines a resolvent positive operator on the space of symmetric matrices; see Appendix A. This means that the covariance function of a suitable Black Scholes model dominates the one of a Heston model in case the volatility function is bounded.

Remark 2. We can use the same approach if we allow for a different volatility $v_{i}(i=$ $1, \ldots, q)$ in every summand of the diffusion in (2.1a). Then, boundedness has to be understood in a more general sense, i.e., we need the existence of a positive semidefinite matrix $\mathbf{C}=$ $\left(c_{i j}\right)_{i, j=1, \ldots, q}$ such that

$$
\left(v_{1}^{\frac{1}{2}}(t), \ldots, v_{q}^{\frac{1}{2}}(t)\right)^{T}\left(v_{1}^{\frac{1}{2}}(t), \ldots, v_{q}^{\frac{1}{2}}(t)\right) \leq \mathbf{C}
$$

for all $t \in[0, T]$. The operator $L$ in Lemma 2.1 then becomes $L(X)=A X+X A^{T}+$ $\sum_{i, j=1}^{q} N_{i} X N_{j}^{T} c_{i j} k_{i j}$. The associated Black Scholes model that guarantees the identity as in Lemma 2.2 is given by setting $v_{i} \equiv 1$ and replacing the Wiener process with covariance matrix $\mathbf{K}$ by a Wiener process with covariance $\mathbf{K} \circ \mathbf{C}$, where $\cdot \circ \cdot$ denotes the component-wise product of two matrices. Notice that $\mathbf{K} \circ \mathbf{C}$ is positive semidefinite again due to Schur's product theorem [40].

3. Characterization of dominant states. We are interested in the dominant subspace of system (2.1) meaning that we aim to identify states that are less important in both (2.1a) and $(2.1 \mathrm{~b})$. Those can be neglected in the system dynamics, leading us to an approximation of the system in a lower dimension.

The objects that we choose to identify unimportant states are related to matrices that are used in deterministic control theory. In linear deterministic control systems, the so-called reachability Gramian characterizes the minimal energy that is needed to steer a system from zero to some desired state at time $T$. Moreover, the observability Gramian determines the energy that is caused by the observations of an unknown initial state on the time interval $[0, T]$; see, e.g., [1]. Consequently, these Gramians can be used to identify states that require a large amount of energy to be reached and states that produce only very little observation energy. Those are less relevant in a control system.

We use these ideas and extend them to the stochastic uncontrolled framework considered here. The matrices identifying the dominant subspaces of system (2.1) will also be called Gramians due to the link between the concepts.

3.1. Dominant subspaces of (2.1a). We introduce the fundamental solution to (2.1a) as an $\mathbb{R}^{n \times n}$-valued stochastic process $\Phi$ solving

Copyright ( by SIAM. Unauthorized reproduction of this article is prohibited. 


$$
\Phi(t)=I+\int_{0}^{t} A \Phi(s) d s+\sum_{i=1}^{q} \int_{0}^{t} \sqrt{v(s)} N_{i} \Phi(s) d W_{i}(s), \quad t \in[0, T],
$$

where $I$ denotes the identity matrix. If $v \equiv c$, the fundamental solution is denoted by $\Phi_{c}$. It is not hard to see that the solution to (2.1a) is given by

$$
x\left(t ; x_{0}\right)=\Phi(t) x_{0}=\Phi(t) B z,
$$

because we assumed that the initial state is spanned by the columns of $B$. Below, $\langle\cdot, \cdot\rangle_{2}$ denotes the Euclidean inner product and $\|\cdot\|_{2}^{2}$ is the corresponding norm.

Based on (3.2), let us now identify the states in (2.1a) that play a minor role. We obtain

$$
\begin{aligned}
\mathbb{E}\left|\left\langle x\left(t ; x_{0}\right), \tilde{x}\right\rangle_{2}\right|^{2} & =\mathbb{E}\left|\langle\Phi(t) B z, \tilde{x}\rangle_{2}\right|^{2}=\mathbb{E}\left|\left\langle z, B^{T} \Phi^{T}(t) \tilde{x}\right\rangle_{2}\right|^{2} \\
& \leq \tilde{x}^{T} \mathbb{E}\left[\Phi(t) B B^{T} \Phi^{T}(t)\right] \tilde{x}\|z\|_{2}^{2}
\end{aligned}
$$

for a given vector $\tilde{x} \in \mathbb{R}^{n}$ and using Cauchy's inequality. Since $\mathbb{E}\left[\Phi(t) B B^{T} \Phi^{T}(t)\right]$ might not be available from the computational point of view, we find an estimate based on $\Phi_{c}$ in the following proposition.

Proposition 3.1. Let $\Phi$ be the fundamental solution to (2.1a), and suppose that $\Phi_{c}$ is the fundamental solution to (2.1a) for the special case $v \equiv c$. Then, we have

$$
\mathbb{E}\left[\Phi(t) B B^{T} \Phi^{T}(t)\right] \leq \mathbb{E}\left[\Phi_{c}(t) B B^{T} \Phi_{c}^{T}(t)\right] .
$$

Proof. We denote the $i$ th column of the matrix $B$ by $b_{i}$, allowing us to write $\Phi(t) B=$ $\left[x\left(t ; b_{1}\right), \ldots, x\left(t ; b_{m}\right)\right]$. Hence, we have

$$
\mathbb{E}\left[\Phi(t) B B^{T} \Phi^{T}(t)\right]=\sum_{k=1}^{m} \mathbb{E}\left[x\left(t ; b_{k}\right) x^{T}\left(t ; b_{k}\right)\right] .
$$

Applying (2.6) to (3.4) yields

$$
\mathbb{E}\left[\Phi(t) B B^{T} \Phi^{T}(t)\right] \leq \sum_{k=1}^{m} \mathbb{E}\left[x_{c}\left(t ; b_{k}\right) x_{c}^{T}\left(t ; b_{k}\right)\right]=\mathbb{E}\left[\Phi_{c}(t) B B^{T} \Phi_{c}^{T}(t)\right] .
$$

This concludes the proof.

Combining (3.3) with Proposition 3.1, we find

$$
\mathbb{E}\left|\left\langle x\left(t ; x_{0}\right), \tilde{x}\right\rangle_{2}\right|^{2} \leq \tilde{x}^{T} F(t) \tilde{x}\|z\|_{2}^{2},
$$

where $F(t):=\mathbb{E}\left[\Phi_{c}(t) B B^{T} \Phi_{c}^{T}(t)\right]$. We define $P_{T}:=\int_{0}^{T} F(t) d t$ and call $P_{T}$ (time-limited) reachability Gramian. Integrating both sides of (3.5) over $[0, T]$ yields

$$
\int_{0}^{T} \mathbb{E}\left|\left\langle x\left(t ; x_{0}\right), \tilde{x}\right\rangle_{2}\right|^{2} d t \leq \tilde{x}^{T} P_{T} \tilde{x}\|z\|_{2}^{2} .
$$

Consequently, the Gramian $P_{T}$ characterizes the relevant subspaces as we see in the next proposition. 
Proposition 3.2. Let $x\left(\cdot ; x_{0}\right)$ be the solution to (2.1a) with initial state $x_{0}=B z ;$ i.e., it is spanned by the columns of $B$. Then, it holds that

$$
x\left(t ; x_{0}\right) \in \operatorname{im}\left(P_{T}\right) \quad \mathbb{P} \otimes d t \text {-a.s. on } \Omega \times[0, T],
$$

where $\operatorname{im}(\cdot)$ denotes the image of a matrix.

Proof. If $\tilde{x} \in \operatorname{ker}\left(P_{T}\right)$, i.e., $\tilde{x}$ lies in the kernel of $P_{T}$, then the left side of (3.6) is zero, which implies that $\left\langle x\left(t ; x_{0}\right), \tilde{x}\right\rangle_{2}=0 \mathbb{P} \otimes d t$-a.s. Since $P_{T}$ is symmetric positive semidefinite, this yields the claim.

Thus, the states that are not in $\operatorname{im}\left(P_{T}\right)$ are not important in (2.1a). However, it is also important to identify the states that play a minor role. Therefore, we turn our attention to states that lie in $\operatorname{im}\left(P_{T}\right)$ but that are nevertheless less important. We can choose an orthonormal basis of eigenvectors $\left(p_{k}\right)_{k=1, \ldots, n}$ of $P_{T}$ with associated eigenvalues $\left(\lambda_{k}\right)_{k=1, \ldots, n}$. Then, the following representation

$$
x\left(t ; x_{0}\right)=\sum_{k=1}^{n}\left\langle x\left(t ; x_{0}\right), p_{k}\right\rangle_{2} p_{k}
$$

holds. Setting $\tilde{x}=p_{k}$ in (3.6) leads to

$$
\int_{0}^{T} \mathbb{E}\left|\left\langle x\left(t ; x_{0}\right), p_{k}\right\rangle_{2}\right|^{2} d t \leq \lambda_{k}\|z\|_{2}^{2} .
$$

Consequently, $x\left(t ; x_{0}\right)$ is small in the direction of $p_{k}$ if $\lambda_{k}$ is small. Hence, states with a large component in the direction of such a $p_{k}$ are less relevant. This means that that eigenspaces of $P_{T}$ corresponding to small eigenvalues $\lambda_{k}$ play a minor role in the system dynamics.

Remark 3. $P_{T}$ is related to the Gramian used in [6]. However, they choose $\lim _{T \rightarrow \infty} P_{T}$ in some asymptotically stable deterministic setting; i.e., $N_{i}=0$ and $\lambda(A) \subset \mathbb{C}_{-}$, where $\lambda(\cdot)$ denotes the spectrum of a matrix. In the stochastic case the respective stability condition were $\mathbb{E}\left\|x_{c}\left(t ; x_{0}\right)\right\|_{2}^{2} \rightarrow 0$ for $t \rightarrow \infty$ and all initial conditions $x_{0}$ (mean square asymptotic stability); see, e.g., [17, 28, 35]. Stability is not assumed in this paper such that $\lim _{T \rightarrow \infty} P_{T}$ does not exist in general. Moreover, the motivation to use the reachability Gramian $P_{T}$ is different from the motivation given in [6].

We conclude this section by a discussion on how to compute $P_{T}$ which allows to identify redundant information in the system. Using the representation of $F$ in (3.4) and applying Lemma 2.2 to every summand of its right side, we see that $F$ satisfies

$$
\dot{F}(t)=A F(t)+F(t) A^{T}+c \sum_{i, j=1}^{q} N_{i} F(t) N_{j}^{T} k_{i j}, \quad F(0)=B B^{T} .
$$

Integrating both sides of (3.8) yields

$$
F(T)-B B^{T}=A P_{T}+P_{T} A^{T}+c \sum_{i, j=1}^{q} N_{i} P_{T} N_{j}^{T} k_{i j} .
$$


This means that the large-scale generalized Lyapunov equation (3.9) needs to be solved to derive $P_{T}$. This can be done also in a large-scale setting for a given left side. However, the left side of (3.9) depends on $F(T)$, a matrix that needs to be computed beforehand. For dimensions $n$ of a few hundreds, this can be done directly by vectorizing (3.8). Defining $f(t):=\operatorname{vec}(F(t))$, we then obtain

$$
\dot{f}(t)=\mathcal{K} f(t), \quad f(0)=\operatorname{vec}\left(B B^{T}\right),
$$

where $\operatorname{vec}(\cdot)$ is the vectorization of a matrix, $\cdot \otimes \cdot$ denotes the Kronecker product of two matrices, and

$$
\mathcal{K}:=I \otimes A+A \otimes I+c \sum_{i, j=1}^{q} N_{i} \otimes N_{j} k_{i j} .
$$

Consequently, deriving $F(T)$ relies on the efficient computation of a matrix exponential, since

$$
f(T)=\mathrm{e}^{\mathcal{K} T} \operatorname{vec}\left(B B^{T}\right) .
$$

A discussion on how to determine a matrix exponential efficiently can be found in [31] and references therein. However, we need to assume that $0 \notin \lambda(\mathcal{K})$. This guarantees a unique solution of (3.9) which we suppose to have below. If system (2.1) were mean square asymptotically stable in the spirit of Remark 3, then we could take $T \rightarrow \infty$ in (3.9) and $F(T)$ would disappear in the limit which makes the computation of the (infinite) reachability Gramian much simpler. Such type of (infinite) reachability Gramians are, e.g., used to characterize reachability energies in mean square asymptotically stable controlled stochastic systems having time-invariant coefficients $[13,35]$, a setting that differs significantly from the one considered here.

More advanced approaches need to be used to solve for $F(T)$ if $n$ is very large. There are relevant examples in which $F(T)$ and hence $P_{T}$ can be derived explicitly as we will see in section 6 .

We give some insight on the computational complexity of the solution to the Lyapunov equation in the following remark.

Remark 4. Once we have determined $F(T)$, a large scale generalized Lyapunov equation (3.9) needs to be solved. If one uses vectorization and the Kronecker product to solve the matrix equation, which then becomes a linear system of size $n^{2}$, then in general $\mathcal{O}\left(n^{6}\right)$ operations are needed to solve this linear system.

However, in the past decades, several techniques have been developed in order to solve the large-scale generalized Lyapunov equation (3.9) more efficiently. Damm [18] has shown that one can compute the solution to (3.9) by solving a sequence of standard Lyapunov equations, that is, the case where $N_{i}=0$ for $i=1, \ldots, q$. Such standard Lyapunov equations can either be solved by direct methods, such as Bartels-Stewart [3], which cost $\mathcal{O}\left(n^{3}\right)$ operations, or by iterative methods such as alternating directions implicit or Krylov subspace methods [42], which have a much smaller complexity than the Bartels-Stewart algorithm, in particular, when the left-hand side is of low rank or structured. In addition, several low rank solvers have been developed for computing the solution to (3.9) directly, for the case where one can show that the solution is approximately of low rank $[8,30,41]$. The complexity of those methods is generally $\mathcal{O}\left(n^{2}\right)$ or less.

Copyright (c) by SIAM. Unauthorized reproduction of this article is prohibited. 
3.2. Dominant subspace of (2.1b). We now characterize the importance of an initial state $x_{0}$ in the output equation. The initial condition is not relevant if the corresponding output $y\left(\cdot ; x_{0}\right)$ has zero energy and is of low relevance if the output energy is small, since those initial states barely contribute to the quantity of interest. We begin with an estimate for $y$ based on the result of section 2 .

Proposition 3.3. Suppose that $y$ is given by (2.1b), and let us assume that $y_{c}$ is the output associated with the solution to the solution of (2.1a) if $v \equiv c$, i.e., $y_{c}(t)=C x_{c}(t)$. Then, we have

$$
\mathbb{E} \int_{0}^{T}\|y(t)\|_{2}^{2} d t \leq \mathbb{E} \int_{0}^{T}\left\|y_{c}(t)\right\|_{2}^{2} d t
$$

Proof. We use the linearity of the trace to obtain

$$
\mathbb{E} \int_{0}^{T}\|y(t)\|_{2}^{2} d t=\mathbb{E} \int_{0}^{T} \operatorname{tr}\left(C x(t) x^{T}(t) C^{T}\right) d t=\int_{0}^{T} \operatorname{tr}\left(C \mathbb{E}\left[x(t) x^{T}(t)\right] C^{T}\right) d t .
$$

Using that (2.6) is preserved when the trace is applied yields

$$
\mathbb{E} \int_{0}^{T}\|y(t)\|_{2}^{2} d t \leq \int_{0}^{T} \operatorname{tr}\left(C \mathbb{E}\left[x_{c}(t) x_{c}^{T}(t)\right] C^{T}\right) d t=\mathbb{E} \int_{0}^{T}\left\|y_{c}(t)\right\|_{2}^{2} d t,
$$

which concludes the proof of this proposition.

Now, the goal is to find a bound for the energy of $y_{c}$. Therefore, we introduce $Q_{T}$ as the solution to

$$
G(T)-C^{T} C=A^{T} Q_{T}+Q_{T} A+c \sum_{i, j=1}^{q} N_{i}^{T} Q_{T} N_{j} k_{i j},
$$

an equation that can be solved for large $n$ once the left side is given. We refer to $Q_{T}$ as the observability Gramian since it characterizes the observation energy as we will see below. $G(t)$, $t \in[0, T]$, entering in (3.13) satisfies

$$
\dot{G}(t)=A^{T} G(t)+G(t) A+c \sum_{i, j=1}^{q} N_{i}^{T} G(t) N_{j} k_{i j}, \quad G(0)=C^{T} C,
$$

i.e., $Q_{T}=\int_{0}^{T} G(s) d s$. Notice that if $n$ is not too large, $G(T)$ can be computed analogously to (3.11) meaning that $g(T):=\operatorname{vec}(G(T))$ is given by

$$
g(T)=\mathrm{e}^{\mathcal{K}^{T} T} \operatorname{vec}\left(C^{T} C\right) .
$$

Below, we distinguish between two cases. We first discuss the case in which the system matrices commute. 
Commuting matrices. We find a representation for $Q_{T}$ and subsequently an energy estimate for $y_{c}$ in case all the matrices in (2.1a) commute. For that purpose, we establish the following result.

Proposition 3.4. Let us assume that all matrices $A, N_{1}, \ldots, N_{q}$ commute. Hence, we have that these matrices commute with the fundamental solution $\Phi_{c}$, i.e.,

$$
A \Phi_{c}(t)=\Phi_{c}(t) A \quad \text { and } \quad N_{i} \Phi_{c}(t)=\Phi_{c}(t) N_{i}
$$

for all $t \in[0, T]$ and $i=1, \ldots, q$.

Proof. These identities hold since the left and the right sides satisfy the same differential equation, e.g., one can multiply (3.1) with $A$ from the left to obtain the equation for $A \Phi_{c}$ and with $A$ from the right to get the one for $\Phi_{c} A$. Since all system matrices commute, the equations coincide. Similarly, one finds the indents for the matrices $N_{i}$.

The example considered in section 6 satisfies the assumption of Proposition 3.4. Furthermore, notice that in the deterministic case $\left(N_{i}=0\right)$ we have $\Phi_{c}(t)=\mathrm{e}^{A t}$ and hence (3.15) is always given. Based on Proposition 3.4, a representation of $Q_{T}$ can be found.

Proposition 3.5. Under the assumptions of Proposition 3.4, we have

$$
Q_{T}=\int_{0}^{T} \mathbb{E}\left[\Phi_{c}^{T}(t) C^{T} C \Phi_{c}(t)\right] d t
$$

Proof. We apply Ito's product rule to $\Phi_{c}^{T}(t) C^{T} C \Phi_{c}(t)$ and take the correlation of the noise processes into account. This yields

$$
\begin{aligned}
d & \left(\Phi_{c}^{T}(t) C^{T} C \Phi_{c}(t)\right) \\
& =d\left(\Phi_{c}^{T}(t)\right) C^{T} C \Phi_{c}(t)+\Phi_{c}^{T}(t) C^{T} C d\left(\Phi_{c}(t)\right)+d\left(\Phi_{c}^{T}(t)\right) C^{T} C d\left(\Phi_{c}(t)\right) \\
& =d\left(\Phi_{c}^{T}(t)\right) C^{T} C \Phi_{c}(t)+\Phi_{c}^{T}(t) C^{T} C d\left(\Phi_{c}(t)\right)+c \sum_{i, j=1}^{q} \Phi_{c}^{T}(t) N_{i}^{T} C^{T} C N_{j} k_{i j} \Phi_{c}(t) d t .
\end{aligned}
$$

Above, we plug in (3.1) for the case when $v \equiv c$ and take the expected value on both sides. Hence, using that the Ito integral has mean zero, we have

$$
\begin{aligned}
& d\left(\mathbb{E}\left[\Phi_{c}^{T}(t) C^{T} C \Phi_{c}(t)\right]\right) \\
& =\mathbb{E}\left[\Phi_{c}^{T}(t)\left(A^{T} C^{T} C+C^{T} C A+c \sum_{i, j=1}^{q} N_{i}^{T} C^{T} C N_{j} k_{i j}\right) \Phi_{c}(t)\right] d t .
\end{aligned}
$$

Due to (3.15) we see that $\mathbb{E}\left[\Phi_{c}^{T}(t) C^{T} C \Phi_{c}(t)\right], t \in[0, T]$, solves (3.14) and thus $Q_{T}=$ $\int_{0}^{T} \mathbb{E}\left[\Phi_{c}^{T}(t) C^{T} C \Phi_{c}(t)\right] d t$.

Inequality (3.12) and Proposition 3.5 now imply that

$$
\mathbb{E} \int_{0}^{T}\|y(t)\|_{2}^{2} d t \leq \mathbb{E} \int_{0}^{T}\left\|C \Phi_{c}(t) x_{0}\right\|_{2}^{2} d t=x_{0}^{T} Q_{T} x_{0} .
$$

Initial states that are spanned by eigenvectors of $Q_{T}$ belonging to the small eigenvalues lead to a small right side in (3.16) and consequently yield a small output $y$. Hence, we know that eigenspaces of $Q_{T}$ corresponding to the small eigenvalues are less relevant in (2.1b).

Copyright (C) by SIAM. Unauthorized reproduction of this article is prohibited. 
General case. We find another bound on the energy of $y_{c}$ and hence also for $y$ in the general case.

Proposition 3.6. If $y$ is the quantity of interest in system (2.1) and $Q_{T}$ the solution of (3.13), then

$$
\mathbb{E} \int_{0}^{T}\|y(t)\|_{2}^{2} d t \leq x_{0}^{T} Q_{T} x_{0}+\mathcal{R}(T)
$$

where $\mathcal{R}(T):=\mathbb{E} \int_{0}^{T} x_{c}^{T}\left(t ; x_{0}\right) G(T) x_{c}\left(t ; x_{0}\right) d t-\mathbb{E}\left[x_{c}^{T}\left(T ; x_{0}\right) Q_{T} x_{c}\left(T ; x_{0}\right)\right]$ with $G$ solving $(3.14)$.

Proof. We make use of

$$
\mathbb{E}\left[x_{c}^{T}\left(T ; x_{0}\right) Q_{T} x_{c}\left(T ; x_{0}\right)\right]=\operatorname{tr}\left(Q_{T} \mathbb{E}\left[x_{c}\left(T ; x_{0}\right) x_{c}^{T}\left(T ; x_{0}\right)\right]\right) .
$$

We obtain from Lemma 2.2 that

$$
\begin{aligned}
Q_{T} \mathbb{E}\left[x_{c}\left(T ; x_{0}\right) x_{c}^{T}\left(T ; x_{0}\right)\right]= & Q_{T} x_{0} x_{0}^{T}+\mathbb{E} \int_{0}^{T} Q_{T} A x_{c}\left(t ; x_{0}\right) x_{c}^{T}\left(t ; x_{0}\right) d t \\
& +\mathbb{E} \int_{0}^{T} Q_{T} x_{c}\left(t ; x_{0}\right) x_{c}^{T}\left(t ; x_{0}\right) A^{T} d t \\
& +c \sum_{i, j=1}^{q} \mathbb{E} \int_{0}^{T} Q_{T} N_{i} x_{c}\left(t ; x_{0}\right) x_{c}^{T}\left(t ; x_{0}\right) N_{j}^{T} k_{i j} d t
\end{aligned}
$$

Using properties of the trace, (3.18) becomes

$$
\begin{aligned}
\mathbb{E} & {\left[x_{c}^{T}\left(T ; x_{0}\right) Q_{T} x_{c}\left(T ; x_{0}\right)\right] } \\
& =x_{0}^{T} Q_{T} x_{0}+\mathbb{E} \int_{0}^{T} x_{c}^{T}\left(t ; x_{0}\right)\left(A^{T} Q_{T}+Q_{T} A+c \sum_{i, j=1}^{q} N_{i}^{T} Q_{T} N_{j} q_{i j}\right) x_{c}\left(t ; x_{0}\right) d s .
\end{aligned}
$$

We insert (3.13) into the above identity to get

$$
\begin{aligned}
\mathbb{E} & {\left[x_{c}^{T}\left(T ; x_{0}\right) Q_{T} x_{c}\left(T ; x_{0}\right)\right]=x_{0}^{T} Q_{T} x_{0}+\mathbb{E} \int_{0}^{T} x_{c}^{T}\left(t ; x_{0}\right)\left(G(T)-C^{T} C\right) x_{c}\left(t ; x_{0}\right) d t } \\
& =x_{0}^{T} Q_{T} x_{0}-\mathbb{E} \int_{0}^{T}\left\|y_{c}(t)\right\|_{2}^{2} d t+\mathbb{E} \int_{0}^{T} x_{c}^{T}\left(t ; x_{0}\right) G(T) x_{c}\left(t ; x_{0}\right) d t .
\end{aligned}
$$

This, together with (3.12), gives us the result.

Assuming that the remainder term $\mathcal{R}(T)$ is not too large, the same conclusions as below (3.16) can be made. The eigenspaces that belong to the small eigenvalues of $Q_{T}$ are unimportant. If the system is mean square asymptotic stable, then $Q_{\infty}:=\lim _{T \rightarrow \infty} Q_{T}$ exists and $\mathcal{R}(T) \rightarrow 0$ as $T \rightarrow \infty$. Taking the limit of $T \rightarrow \infty$ in (3.17) would then lead to a characterization of the output energy by $Q_{\infty}$ without a remainder term. $Q_{\infty}$ is also easier to determine than $Q_{T}$ since it solves (3.13) with $G(T)=0$. Energy estimates based on $Q_{\infty}$ are shown in $[9,13,35]$ if the variance $v$ is constant. 
We can also get to a more explicit bound by applying Gronwall's lemma to (3.19) if $Q_{T}$ is regular. Defining $\alpha(T):=x_{0}^{T} Q_{T} x_{0}-\mathbb{E} \int_{0}^{T}\left\|y_{c}(t)\right\|_{2}^{2} d t$ (3.19) becomes

$$
\begin{aligned}
\mathbb{E}\left[\left\|Q_{T}^{\frac{1}{2}} x_{c}\left(t ; x_{0}\right)\right\|_{2}^{2}\right] & =\alpha(T)+\mathbb{E} \int_{0}^{T}\left\|G^{\frac{1}{2}}(T) x_{c}\left(t ; x_{0}\right)\right\|_{2}^{2} d t \\
& \leq \alpha(T)+k_{T} \mathbb{E} \int_{0}^{T}\left\|Q_{T}^{\frac{1}{2}} x_{c}\left(t ; x_{0}\right)\right\|_{2}^{2} d t
\end{aligned}
$$

where $k_{T}:=\left\|G^{\frac{1}{2}}(T) Q_{T}^{-\frac{1}{2}}\right\|_{2}^{2}$. Gronwall's lemma leads to

$$
0 \leq \mathbb{E}\left[\left\|Q_{T}^{\frac{1}{2}} x_{c}\left(t ; x_{0}\right)\right\|_{2}^{2}\right] \leq \alpha(T)+\mathbb{E} \int_{0}^{T} \alpha(t) k_{T} \mathrm{e}^{k_{T}(T-t)} d t .
$$

With a few more steps, we find

$$
\mathbb{E} \int_{0}^{T}\|y(t)\|_{2}^{2} d t \leq x_{0}^{T} Q_{T} x_{0} \mathrm{e}^{k_{T} T},
$$

but this bound cannot be expected to be tight.

4. State-space transformation and reduced-order model. Balancing related MOR like balanced truncation were initially invented for controlled linear deterministic systems that are asymptotically stable and have zero initial states [33]. Balanced truncation has been extended to stochastic systems with similar properties [9, 13]. Subsequently, this scheme was studied for deterministic and stochastic systems with nonzero initial conditions [6, 7]. However, all these methods are restricted to stable systems. A method for deterministic equations called time-limited balanced truncation aiming to create a good reduced system on a finite time interval only was introduced in [22]. As pointed out in [31], this method has some potential in the context of unstable systems.

The method explained below is a combination of all the methods mentioned above. It follows the same concept which is simultaneously diagonalizing system Gramians. Here, the Gramians are $P_{T}$ and $Q_{T}$ solving (3.9) and (3.13), respectively. We have shown the relevance of these Gramians in section 3. Diagonalizing both $P_{T}$ and $Q_{T}$ means that we create a system in which the important states in (2.1a) and (2.1b) are the same. Hence, the unimportant ones can be easily identified and thus truncated.

Let $S \in \mathbb{R}^{n \times n}$ be a regular matrix. We do a coordinate transformation by introducing

$$
\hat{x}(t)=S x(t) .
$$

Based on (2.1) the associated system is

$$
\begin{aligned}
d \hat{x}(t) & =\hat{A} \hat{x}(t) d t+\sum_{i=1}^{q} \sqrt{v(t)} \hat{N}_{i} x(t) d W_{i}(t), \quad \hat{x}(0)=S x_{0}=S B z, \\
y(t) & =\hat{C} \hat{x}(t), \quad t \in[0, T],
\end{aligned}
$$

Copyright (C) by SIAM. Unauthorized reproduction of this article is prohibited. 
where $\hat{A}=S A S^{-1}, \hat{B}=S B, \hat{C}=C S^{-1}$, and $\hat{N}_{i}=S N_{i} S^{-1}$. Notice that the quantity of interest does not change with this transformation. However, the matrices characterizing the importance of states in (4.1a) and (4.1b) are different ones. For the transformed system (4.1), these become

$$
\hat{P}_{T}=S P_{T} S^{T} \quad \text { and } \quad \hat{Q}_{T}=S^{-T} Q_{T} S^{-1} .
$$

The above relation is obtained by multiplying (3.8) with $S$ from the left and with $S^{T}$. Moreover, (3.14) needs to be multiplied with $S^{-T}$ from the left and with $S^{-1}$ from the right.

We now choose $S$ such that $\hat{P}_{T}=\hat{Q}_{T}=\Sigma_{T}=\operatorname{diag}\left(\sigma_{1}, \ldots, \sigma_{n}\right)$, where $\sigma_{1} \geq \cdots \geq \sigma_{n}>0$ are called Hankel singular values (HSVs) and given by $\sigma_{i}=\sqrt{\lambda_{i}\left(P_{T} Q_{T}\right)}$, where $\lambda_{i}(\cdot)$ denotes the $i$ th eigenvalue of the matrix and $i=1, \ldots, n$. Such a system is called balanced. A transformation like this always exists if $P_{T}, Q_{T}>0$. It is, together with its inverse, derived the following way:

$$
S=\Sigma_{T}^{-\frac{1}{2}} U^{T} L_{Q}^{T} \quad \text { and } \quad S^{-1}=K_{P} V \Sigma_{T}^{-\frac{1}{2}} .
$$

The above matrices are computed from factorizations $P_{T}=K_{P} K_{P}^{T}$ and $Q_{T}=L_{Q} L_{Q}^{T}$ as well as from the singular value decomposition of $K_{P}^{T} L_{Q}=V \Sigma U^{T}$.

In a balanced system, it is easy to identify the unimportant states. They are the ones corresponding to the small HSVs of the system and represented by $x_{2}$ given by the partition of the balanced state variable

$$
\hat{x}(t)=S x(t)=\left[\begin{array}{l}
x_{1}(t) \\
x_{2}(t)
\end{array}\right],
$$

where $x_{1}(t) \in \mathbb{R}^{\tilde{n}}$ represents the relevant states in the system dynamics. Furthermore, we partition the balanced realization as follows:

$$
S A S^{-1}=\left[\begin{array}{ll}
A_{11} & A_{12} \\
A_{21} & A_{22}
\end{array}\right], \quad S B=\left[\begin{array}{c}
B_{1} \\
B_{2}
\end{array}\right], \quad C S^{-1}=\left[\begin{array}{ll}
C_{1} & C_{2}
\end{array}\right], \quad S N_{i} S^{-1}=\left[\begin{array}{ll}
N_{i, 11} & N_{i, 12} \\
N_{i, 21} & N_{i, 22}
\end{array}\right],
$$

where $A_{11} \in \mathbb{R}^{\tilde{n} \times \tilde{n}}$ and so forth. With this, system (4.1) becomes

$$
\begin{aligned}
{\left[\begin{array}{l}
d x_{1} \\
d x_{2}
\end{array}\right] } & =\left[\begin{array}{ll}
A_{11} & A_{12} \\
A_{21} & A_{22}
\end{array}\right]\left[\begin{array}{l}
x_{1} \\
x_{2}
\end{array}\right] d t+\sum_{i=1}^{q} \sqrt{v}\left[\begin{array}{ll}
N_{i, 11} & N_{i, 12} \\
N_{i, 21} & N_{i, 22}
\end{array}\right]\left[\begin{array}{l}
x_{1} \\
x_{2}
\end{array}\right] d W_{i}, \quad \hat{x}(0)=\left[\begin{array}{l}
B_{1} \\
B_{2}
\end{array}\right] z, \\
y(t) & =\left[\begin{array}{ll}
C_{1} & C_{2}
\end{array}\right]\left[\begin{array}{l}
x_{1}(t) \\
x_{2}(t)
\end{array}\right], \quad t \in[0, T] .
\end{aligned}
$$

The time dependence is omitted in (4.2) to shorten the notation. The reduced system of dimension $\tilde{n} \ll n$ is now obtained by neglecting $x_{2}$; i.e., the second line in (4.2) is truncated the remaining $x_{2}$ variables are set zero in both the first line of (4.2) and in (4.3). The reducedorder model then is

$$
\begin{aligned}
d \tilde{x}(t) & =A_{11} \tilde{x}(t) d t+\sum_{i=1}^{q} \sqrt{v(t)} N_{i, 11} \tilde{x}(t) d W_{i}(t), \quad \tilde{x}(0)=B_{1} z, \\
\tilde{y}(t) & =C_{1} \tilde{x}(t), \quad t \in[0, T]
\end{aligned}
$$

where $A_{11}, N_{i, 11} \in \mathbb{R}^{\tilde{n} \times \tilde{n}}, B_{1} \in \mathbb{R}^{\tilde{n} \times m}$, and $C_{1} \in \mathbb{R}^{p \times \tilde{n}}$. 
5. Error bound analysis. We introduce an error system by combining (2.1a) and (4.4a) with an output equation that represents the error between (2.1b) and (4.4b). The error system is

$$
\begin{aligned}
d x^{e}(t) & =A^{e} x^{e}(t) d t+\sum_{i=1}^{q} \sqrt{v(t)} N_{i}^{e} x^{e}(t) d W_{i}(t), \quad x^{e}(0)=B^{e} z, \\
y^{e}(t) & =C^{e} x^{e}(t), \quad t \in[0, T],
\end{aligned}
$$

where the error state $x^{e}$ and the error matrices $\left(A^{e}, B^{e}, C^{e}, N_{i}^{e}\right)$ are

$$
x^{e}=\left[\begin{array}{l}
x \\
\tilde{x}
\end{array}\right], A^{e}=\left[\begin{array}{cc}
A & 0 \\
0 & A_{11}
\end{array}\right], B^{e}=\left[\begin{array}{c}
B \\
B_{1}
\end{array}\right], C^{e}=\left[\begin{array}{ll}
C & -C_{1}
\end{array}\right], N_{i}^{e}=\left[\begin{array}{cc}
N_{i} & 0 \\
0 & N_{i, 11}
\end{array}\right] .
$$

Let us again assume that an index $c$ indicates that $v$ in (5.1) is replaced by $c$. We obtain

$$
\mathbb{E} \int_{0}^{T}\left\|y^{e}(t)\right\|_{2}^{2} d t \leq \mathbb{E} \int_{0}^{T}\left\|y_{c}^{e}(t)\right\|_{2}^{2} d t
$$

the same way as in (3.12). Based on the fundamental solution $\Phi^{e}$ (or $\Phi_{c}^{e}$ ), the quantity of interest given a constant volatility function is represented by $y_{c}^{e}(t)=C^{e} \Phi_{c}^{e}(t) B^{e} z$. Plugging this into the above inequality yields

$$
\begin{aligned}
\mathbb{E} \int_{0}^{T}\left\|y^{e}(t)\right\|_{2}^{2} d t & \leq \mathbb{E} \int_{0}^{T}\left\|C^{e} \Phi_{c}^{e}(t) B^{e} z\right\|_{2}^{2} d t \leq \mathbb{E} \int_{0}^{T}\left\|C^{e} \Phi_{c}^{e}(t) B^{e}\right\|_{F}^{2} d t\|z\|_{2}^{2} \\
& =\operatorname{tr}\left(C^{e} P_{T}^{e}\left(C^{e}\right)^{T}\right)\|z\|_{2}^{2}
\end{aligned}
$$

where we set $P_{T}^{e}:=\int_{0}^{T} F^{e}(t) d t$ with $F^{e}(t)=\Phi_{c}^{e}(t) B^{e}\left(B^{e}\right)^{T}\left(\Phi_{c}^{e}\right)^{T}(t)$. Analogue to (3.8), $F^{e}$ solves

$$
\dot{F}^{e}(t)=A^{e} F^{e}(t)+F^{e}(t)\left(A^{e}\right)^{T}+c \sum_{i, j=1}^{q} N_{i}^{e} F^{e}(t)\left(N_{j}^{e}\right)^{T} k_{i j}, \quad F^{e}(0)=B^{e}\left(B^{e}\right)^{T} .
$$

We partition the solution to (5.4) as follows

$$
F^{e}(t)=\left[\begin{array}{ll}
F_{11}(t) & F_{12}(t) \\
F_{12}^{T}(t) & F_{22}(t)
\end{array}\right]
$$

and see that $F_{11}(t)=F(t)$ solving $(3.8)$ as well as $F_{12}(t)=\bar{F}(t)$ and $F_{22}(t)=\tilde{F}(t)$ that are the solutions to

$$
\begin{aligned}
& \dot{\bar{F}}(t)=A \bar{F}(t)+\bar{F}(t) A_{11}^{T}+c \sum_{i, j=1}^{q} N_{i} \bar{F}(t) N_{j, 11}^{T} k_{i j}, \quad \bar{F}(0)=B B_{1}^{T}, \\
& \dot{\tilde{F}}(t)=A_{11} \tilde{F}(t)+\tilde{F}(t) A_{11}^{T}+c \sum_{i, j=1}^{q} N_{i, 11} \tilde{F}(t) N_{j, 11}^{T} k_{i j}, \quad \tilde{F}(0)=B_{1} B_{1}^{T},
\end{aligned}
$$

using the partitions in (5.2). From (5.3) and (5.5), we obtain 


$$
\begin{aligned}
\mathbb{E} \int_{0}^{T}\|y(t)-\tilde{y}(t)\|_{2}^{2} d t & =\mathbb{E} \int_{0}^{T}\left\|y^{e}(t)\right\|_{2}^{2} d t \\
& \leq\left(\operatorname{tr}\left(C P_{T} C^{T}\right)-2 \operatorname{tr}\left(C \bar{P}_{T} C_{1}^{T}\right)+\operatorname{tr}\left(C_{1} \tilde{P}_{T} C_{1}^{T}\right)\right)\|z\|_{2}^{2},
\end{aligned}
$$

where $\bar{P}_{T}:=\int_{0}^{T} \bar{F}(t) d t$ and $\tilde{P}_{T}:=\int_{0}^{T} \tilde{F}(t) d t$. By integrating both (5.6) and (5.7), the equations for these two matrices are

$$
\begin{gathered}
\bar{F}(T)-B B_{1}^{T}=A \bar{P}_{T}+\bar{P}_{T} A_{11}^{T}+c \sum_{i, j=1}^{q} N_{i} \bar{P}_{T} N_{j, 11}^{T} k_{i j}, \\
\tilde{F}(T)-B_{1} B_{1}^{T}=A_{11} \tilde{P}_{T}+\tilde{P}_{T} A_{11}^{T}+c \sum_{i, j=1}^{q} N_{i, 11} \tilde{P}_{T} N_{j, 11}^{T} k_{i j} .
\end{gathered}
$$

Since $P_{T}$ is already known from the balancing procedure explained in section 4 , the bound for the absolute output error in (5.8) requires only the computation of $\bar{P}_{T}$ and $\tilde{P}_{T}$. Since the reduced dimension $\tilde{n}$ is rather small, the corresponding equations (5.9) and (5.10) can often be solved directly through vectorization. Hence, we have

$$
\begin{aligned}
\operatorname{vec}(\bar{F}(T))-\operatorname{vec}\left(B B_{1}^{T}\right) & =\overline{\mathcal{K}} \operatorname{vec}\left(\bar{P}_{T}\right), \\
\operatorname{vec}(\tilde{F}(T))-\operatorname{vec}\left(B_{1} B_{1}^{T}\right) & =\tilde{\mathcal{K}} \operatorname{vec}\left(\tilde{P}_{T}\right),
\end{aligned}
$$

with $\bar{F}(T)=\mathrm{e}^{\overline{\mathcal{K}} T} \operatorname{vec}\left(B B_{1}^{T}\right)$ and $\tilde{F}(T)=\mathrm{e}^{\tilde{\mathcal{K}} T} \operatorname{vec}\left(B_{1} B_{1}^{T}\right)$, where

$$
\begin{aligned}
& \overline{\mathcal{K}}:=\left(I_{n} \otimes A_{11}+A \otimes I_{\tilde{n}}+c \sum_{i, j=1}^{q} N_{i} \otimes N_{j, 11} k_{i j}\right), \\
& \tilde{\mathcal{K}}:=\left(I_{\tilde{n}} \otimes A_{11}+A_{11} \otimes I_{\tilde{n}}+c \sum_{i, j=1}^{q} N_{i, 11} \otimes N_{j, 11} k_{i j}\right) .
\end{aligned}
$$

Above, the identity matrices are equipped with an index indicating the respective dimension. With (5.8) a bound for the absolute error of reducing system (2.1) was found. However, the relative error is more interesting to be analyzed. Therefore, we need a computable lower bound for the $L^{2}$-norm of $y$. This task is relatively simple because the inequality of Cauchy-Schwarz yields

$$
\int_{0}^{T}\|\mathbb{E}[y(t)]\|_{2}^{2} d t \leq \mathbb{E} \int_{0}^{T}\|y(t)\|_{2}^{2} d t .
$$

Now, $\mathbb{E}[x(t)], t \in[0, T]$, solves (2.1a) with $c=0$ which can be seen easily by applying the expected value to both sides of (2.1a) and by exploiting that the Ito integrals have zero mean. We use that $\Phi_{c=0}(t)=\mathrm{e}^{A t}$ such that $\mathbb{E}[y(t)]=C \mathrm{e}^{A t} B z$. We plug this into the above estimate leading to

$$
\mathbb{E} \int_{0}^{T}\|y(t)\|_{2}^{2} d t \geq \int_{0}^{T}\left\|C \mathrm{e}^{A t} B z\right\|_{2}^{2} d t=z^{T} B^{T} Q_{T, 0} B z
$$


where $Q_{T, 0}:=\int_{0}^{T} \mathrm{e}^{A^{T} t} C^{T} C \mathrm{e}^{A t} d t$. According to subsection 3.2, $Q_{T, 0}$ solves (3.13) with $c=0$; i.e.,

$$
\mathrm{e}^{A^{T} T} C^{T} C \mathrm{e}^{A T}-C^{T} C=A^{T} Q_{T, 0}+Q_{T, 0} A,
$$

an equation that can be solved in a large-scale setting, since there are efficient methods to determine $\mathrm{e}^{A T}$ for large $n$. We summarize the results of this section in a theorem below, where we set $\|y\|_{L^{2}}^{2}:=\mathbb{E} \int_{0}^{T}\|y(t)\|_{2}^{2} d t$.

Theorem 5.1. Let $y$ be the output of the original system (2.1), and let $\tilde{y}$ be the output of the reduced model (4.4). Then, the relative $L^{2}$-error between $y$ and $\tilde{y}$ is bounded as follows:

$$
\frac{\|y-\tilde{y}\|_{L^{2}}}{\|y\|_{L^{2}}} \leq \frac{\left(\operatorname{tr}\left(C P_{T} C^{T}\right)-2 \operatorname{tr}\left(C \bar{P}_{T} C_{1}^{T}\right)+\operatorname{tr}\left(C_{1} \tilde{P}_{T} C_{1}^{T}\right)\right)^{\frac{1}{2}}\|z\|_{2}}{\left(z^{T} B^{T} Q_{T, 0} B z\right)^{\frac{1}{2}}},
$$

where $P_{T}, \bar{P}_{T}, \tilde{P}_{T}$, and $Q_{T, 0}$ are the solutions to (3.9), (5.9), (5.10), and (5.12), respectively.

Proof. The result follows from (5.8) and (5.11).

The bound in Theorem 5.1 provides a good a priori error estimate, an indicator for the quality of the reduced system. Error bounds for related methods in a deterministic framework can be found in $[24,37,38]$.

Remark 5. Notice that if we aim to reduce a Black-Scholes model with output $y_{c}$ instead of a Heston model with output $y$, the energy $\left\|y_{c}\right\|_{L^{2}}$ is explicitly known according to section 3.2 given that $A, N_{1}, \ldots, N_{q}$ commute. Then, it holds that $\left\|y_{c}\right\|_{L^{2}}=x_{0}^{T} Q_{T} x_{0}$ such that we can replace $Q_{T, 0}$ by $Q_{T}$ in Theorem 5.1.

Remark 6. For option pricing, we typically consider expectations of $f(y(t))$ for some payoff function $f$. If $f$ is Lipschitz (e.g., for put and call options), then the error bound of Theorem 5.1 immediately carries over. However, there are relevant financial options with even discontinuous payoffs, for instance, digital options. In this case, a general error analysis is difficult. However, we would like to point out that financial models often have inherent smoothing properties, which allow us to effectively mollify the payoff without adding additional bias. We refer to [5] for an application of this property to option pricing with QMC and adaptive sparse grids quadrature methods.

6. Numerical experiments. We apply the MOR technique motivated in section 3 and explained in section 4 . The goal is to accurately approximate payoff functions associated with the large asset price model (2.1a) (these are functions of the quantity of interest in (2.1b)) by payoff functions of the reduced system (4.4). This type of problem is of particular interest if we price European options with an underlying high-dimensional Heston model because computational complexity can be reduced. Moreover, since the reduced system shows good pathwise approximations, it can be of interest in the context of Bermudan options because regression based methods $[32,43]$ suffer from the curse of dimensionality which makes them inaccurate in a large-scale setting. Below, we consider a particular Heston model (2.1) and illustrate the quality of the reduction in dependence of the covariance matrix $\mathbf{K}$ of the noise process.

Copyright (c) by SIAM. Unauthorized reproduction of this article is prohibited. 
We consider the following linear stochastic differential equation that represents an asset price model:

$$
d x_{i}(t)=\mathrm{r} x_{i}(t) d t+\xi_{i} \sqrt{v(t)} x_{i}(t) d W_{i}(t), \quad x_{i}(0)=x_{0, i},
$$

where $x_{i}$ denotes the $i$ th component of a price process $x(i=1, \ldots, n)$. Moreover, we assume that $\mathrm{r}=0.02$ is the fixed interest rate and $\xi_{i} \in[0.2,0.7]$ are volatility parameters sampled from a uniform distribution. Now, we can rewrite (6.1) in order to guarantee the form given in (2.1a). The respective matrices are

$$
A=\mathrm{r} I, \quad N_{i}=\xi_{i} e_{i} e_{i}^{T}, \quad B=x_{0}, \quad \text { and } \quad z=1,
$$

where $e_{i}$ is the $i$ th unit vector in $\mathbb{R}^{n}, q=n$, and assuming that we are interested in a single initial value $x_{0}$ only. In this particular situation, the matrices $A, N_{1}, \ldots, N_{n}$ commute and are symmetric. The variance process is $v(t)=\min \{\bar{v}(t), c\}, t \in[0, T]$, where $\bar{v}$ is the solution to the following stochastic differential equation:

$$
d \bar{v}(t)=a(b-\bar{v}(t)) d t+\bar{\sigma} \sqrt{\bar{v}(t)} d \bar{B}(t), \quad v(0)=v_{0},
$$

where $b=0.2$ is the long run average variance, $a=0.2$ is the rate characterizing the speed of convergence of the average variance, and $\bar{\sigma}=0.15$ is the volatility of the volatility process. Furthermore, $\bar{B}$ is a standard Brownian motion with respect to the filtration $\left(\mathcal{F}_{t}\right)_{t \in[0, T]}$ that negatively correlated with the other standard Brownian motions $W_{i}$; i.e., $\mathbb{E}\left[\bar{B}(t) W_{i}(t)\right]=\rho_{i} t$, where $\rho_{i}<0$. The parameters $\xi_{i}, a, b, \bar{\sigma}$ are chosen to have an average volatility around 0.2 , i.e.,

$$
\mathbb{E}[\sqrt{v(t)}] \frac{1}{n} \sum_{i=1}^{n} \xi_{i} \approx 0.2 .
$$

Notice that in order to fit the theory, the process $v$ is bounded by a constant $c$ since generally $\bar{v}$ is unbounded. Practically, we simulate a certain number of paths of $\bar{v}$ and choose a $c$ that represents a bound of these simulated paths such that those coincide with the respective paths of $v$.

Suppose that the quantity of interest is now some one-dimensional partial information $y$ of the price process $x$ that is the form

$$
y(t)=C x(t),
$$

where the output matrix is $C=[1,1, \ldots 1]$. We now determine a reduced system (4.4). To do so, the matrices $P_{T}$ and $Q_{T}$ need to be computed in order to conduct the balancing procedure described in section 4. Fortunately, these matrices can be derived explicitly from (3.8) and (3.14). Plugging in (6.2) into these equations, we obtain

$$
\begin{aligned}
& \dot{F}(t)=2 \mathrm{r} I F(t)+c \sum_{i, j=1}^{n} e_{i} e_{i}^{T} F(t) e_{j} e_{j}^{T} \xi_{i} \xi_{j} k_{i j}, \quad F(0)=x_{0} x_{0}^{T}, \\
& \dot{G}(t)=2 \mathrm{r} I G(t)+c \sum_{i, j=1}^{n} e_{i} e_{i}^{T} G(t) e_{j} e_{j}^{T} \xi_{i} \xi_{j} k_{i j}, \quad G(0)=C^{T} C .
\end{aligned}
$$

Copyright (C) by SIAM. Unauthorized reproduction of this article is prohibited. 
By multiplying the above equations with $e_{i}^{T}$ from the left and with $e_{j}$ from the right, we can see that these equations can be solved component-wise. The entries of $F(t)=\left(f_{i j}\right)_{i, j=1, \ldots n}$ satisfy

$$
\dot{f}_{i j}(t)=\left(2 \mathrm{r}+c \xi_{i} \xi_{j} k_{i j}\right) f_{i j}(t), \quad f_{i j}(0)=x_{0, i} x_{0, j},
$$

such that $f_{i j}(t)=\mathrm{e}^{h_{i j} t} x_{0, i} x_{0, j}$, where $h_{i j}:=\left(2 \mathrm{r}+c \xi_{i} \xi_{j} k_{i j}\right)$. Integrating $f_{i j}$ over $[0, T]$, we find that $P_{T}=\left(p_{i j}\right)_{i, j=1, \ldots n}$ is given by

$$
p_{i j}=\frac{\mathrm{e}^{h_{i j} T}-1}{h_{i j}} x_{0, i} x_{0, j}
$$

Analogously, it holds that $Q_{T}=\left(q_{i j}\right)_{i, j=1, \ldots n}$ is represented by

$$
q_{i j}=\frac{\mathrm{e}^{h_{i j} T}-1}{h_{i j}} e_{i}^{T} C^{T} C e_{j} .
$$

Since $P_{T}$ and $Q_{T}$ are given explicitly, the reduced system (4.4) with output $\tilde{y}$ comes basically for free in terms of computational time. This also means that we are able to derive a reduced model if the number of assets is very large. We investigate the reduction quality for three different covariance matrices. First we consider a matrix with both small and large correlations between the noise processes. We first choose $\mathbf{K}=\mathbf{K}_{0}:=\tau \tau^{T}$ according to [19], where $\tau=\left[\tau_{1}, \tau_{2}, \ldots \tau_{n}\right]$ has columns $\tau_{i}$ generated by a vector $s=\left(s_{i}\right)_{i=1, \ldots, n-1}$ of samples $s_{i}$ of independent uniformly distributed random variables with values in $[0.8,1]$ :

$$
\tau_{1}=\left(\begin{array}{c}
1 \\
\operatorname{cp}(s)
\end{array}\right), \quad \tau_{2}=\sqrt{1-s_{1}^{2}}\left(\begin{array}{c}
0 \\
1 \\
\operatorname{cp}\left(s_{2: n-1}\right)
\end{array}\right), \ldots, \tau_{n}=\sqrt{1-s_{n-1}^{2}}\left(\begin{array}{c}
0 \\
\vdots \\
0 \\
1
\end{array}\right) .
$$

Above, we set $s_{\ell: n-1}:=\left(s_{\ell}, s_{\ell+1}, \ldots, s_{n-1}\right)^{T}$ and

$$
\operatorname{cp}(s):=\left[s_{1}, s_{1} s_{2}, \ldots, s_{1} s_{2} \ldots s_{n-1}\right]^{T} .
$$

Further, we study the two extreme cases of $\mathbf{K}=I$ (independent noise processes) and $\mathbf{K}=\mathbf{1 1}^{T}$ (perfect correlation), where $\mathbf{1}$ is an $n$-dimensional vector of ones. We choose $n=100, T=1$, $c=0.6$, and the initial conditions $x_{0, i} \in[0,1.25]$ are generated randomly. For $\mathbf{K}=\mathbf{K}_{0}$ we choose $\rho_{i} \in[-0.9,0)$, in case of $\mathbf{K}=I$ we have $\rho_{i}=-0.09$, and we fix $\rho_{i}=-0.5$ for $\mathbf{K}=\mathbf{1 1}^{T}$. In all the numerical experiments below, $2 \mathrm{e} 06$ samples are generated.

6.1. Approximation error in the quantity of interest. We begin with analyzing the error between the output of the full system $y$ and the output of the reduced system $\tilde{y}$. The first question is how to choose the dimension $\tilde{n}$ of system (4.4). The HSVs, i.e., $\sigma_{i}=\sqrt{\lambda_{i}\left(P_{T} Q_{T}\right)}$ are a very good indicator for a suitable choice since the smaller $\sigma_{i}$, the less important the $i$ th state component $\hat{x}_{i}$ in the balanced system (4.1) according to what we have derived in section 3. We can see these values in logarithmic scale for different covariance matrices $\mathbf{K}$ in Figure 6.1. In each case, we observe that one variable dominates the dynamics meaning that no matter how $\mathbf{K}$ is chosen, a scalar reduced-order model already leads to a relatively good approximation. Moreover, we see that the reduction is expected to be least efficient if all noise processes are independent due to a slow decay of the HSVs. However, with perfect correlation $\left(\mathbf{K}=\mathbf{1 1}^{T}\right.$ ), we only have four nonzero HSVs meaning that the 100-dimensional model can be perfectly approximated by a system of four variables. In the case of $\mathbf{K}=\mathbf{K}_{0}$ 
the performance is in between the independent and perfectly correlated scenarios. This fits to our general observation that the higher the correlation, the better the algorithm works.

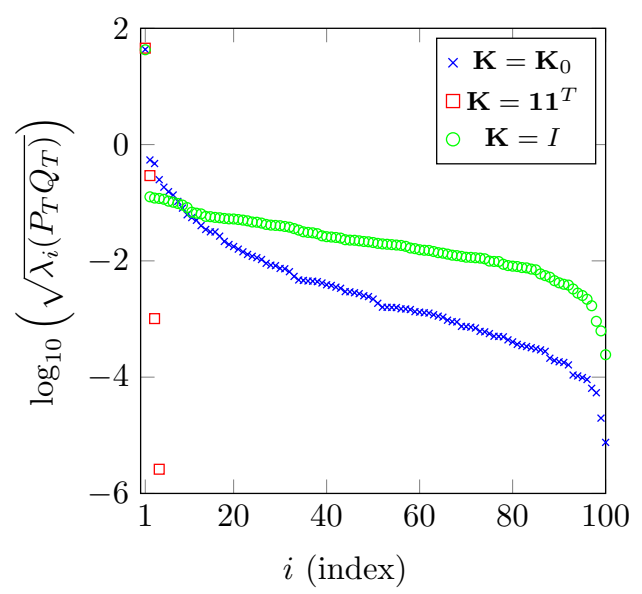

Figure 6.1. Logarithmic HSVs of the large-scale asset model.

We conclude this subsection by a discussion on the error between the outputs $y$ and $\tilde{y}$ of systems (2.1) and (4.4) for this particular example. We determine the relative $L^{2}$-error and the corresponding error bound in Theorem 5.1. This bound that is the right side of (5.13) is denoted by $\mathcal{E} \mathcal{B}$ here. The error bound is relatively tight for the example. In most of the cases it estimates the exact error by factor of three, see Tables 6.1, 6.2, and 6.3. As supposed from the HSVs, very good results are obtained for very small $\tilde{n}$ if $\mathbf{K}=\mathbf{1 1}^{T}$; compare with Figure 6.2. However, in the situation of $\mathbf{K}=\mathbf{K}_{0}$, a reduced-order between 4 and 10 shows a low error, too.

Table 6.1

Relative $L^{2}$-error and error bound for $\mathbf{K}=\mathbf{K}_{0}$.

\begin{tabular}{|c|c|c|}
\hline$\tilde{n}$ & $\|y-\tilde{y}\|_{L^{2}} /\|y\|_{L^{2}}$ & $\mathcal{E} \mathcal{B}$ \\
\hline \hline 1 & $5.10 \mathrm{e}-03$ & $1.55 \mathrm{e}-02$ \\
4 & $2.02 \mathrm{e}-03$ & $6.18 \mathrm{e}-03$ \\
10 & $7.01 \mathrm{e}-04$ & $2.17 \mathrm{e}-03$ \\
15 & $3.95 \mathrm{e}-04$ & $1.23 \mathrm{e}-03$ \\
20 & $2.39 \mathrm{e}-04$ & $7.46 \mathrm{e}-04$ \\
25 & $1.62 \mathrm{e}-04$ & $5.06 \mathrm{e}-04$ \\
50 & $3.56 \mathrm{e}-05$ & $1.13 \mathrm{e}-04$ \\
\hline
\end{tabular}

Table 6.2

Relative $L^{2}$-error and error bound for $\mathbf{K}=I$.

\begin{tabular}{|c|c|c|}
\hline$\tilde{n}$ & $\|y-\tilde{y}\|_{L^{2}} /\|y\|_{L^{2}}$ & $\mathcal{E} \mathcal{B}$ \\
\hline \hline 1 & $2.56 \mathrm{e}-03$ & $7.79 \mathrm{e}-03$ \\
4 & $2.23 \mathrm{e}-03$ & $6.79 \mathrm{e}-03$ \\
10 & $1.69 \mathrm{e}-03$ & $5.12 \mathrm{e}-03$ \\
15 & $1.46 \mathrm{e}-03$ & $4.42 \mathrm{e}-03$ \\
20 & $1.27 \mathrm{e}-03$ & $3.84 \mathrm{e}-03$ \\
25 & $1.08 \mathrm{e}-03$ & $3.28 \mathrm{e}-03$ \\
50 & $5.01 \mathrm{e}-04$ & $1.51 \mathrm{e}-03$ \\
\hline
\end{tabular}

Table 6.3

Relative $L^{2}$-error and error bound for $\mathbf{K}=\mathbf{1 1}^{T}$.

\begin{tabular}{|c|c|c|}
\hline$\tilde{n}$ & $\|y-\tilde{y}\|_{L^{2}} /\|y\|_{L^{2}}$ & $\mathcal{E} \mathcal{B}$ \\
\hline \hline 1 & $2.40 \mathrm{e}-03$ & $5.48 \mathrm{e}-03$ \\
2 & $3.25 \mathrm{e}-06$ & $1.34 \mathrm{e}-05$ \\
3 & $2.94 \mathrm{e}-09$ & $2.98 \mathrm{e}-08$ \\
4 & $3.76 \mathrm{e}-12$ & $2.76 \mathrm{e}-09$ \\
\hline
\end{tabular}

Copyright $@$ by SIAM. Unauthorized reproduction of this article is prohibited. 


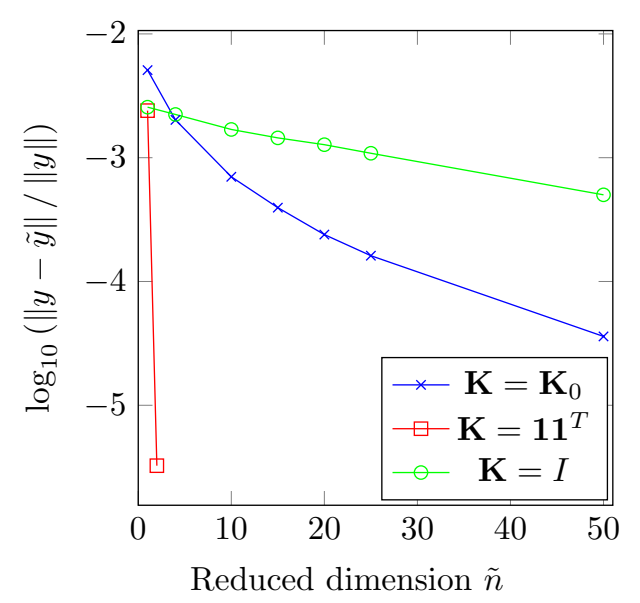

Figure 6.2. Logarithmic relative $L^{2}$-error between $y$ and $\tilde{y}$ for $\tilde{n} \in\{1,4,10,15,20,25,50\}$ if $\mathbf{K}=\mathbf{K}_{0}, I$ and for $\tilde{n}=1,2$ if $\mathbf{K}=\mathbf{1 1}^{T}$.

We conclude this subsection by briefly discussing the robustness of our algorithm in the parameter $\bar{\sigma}$ which is the volatility of the volatility processes $\bar{v}$ given in (6.3) and the bound $c$ that that defines the truncated volatility process $v$. We consider the same framework as above apart from an enlarged volatility parameter which we choose $\bar{\sigma}=0.25$ for the moment. As mentioned above, we practically simulate and use paths of $\bar{v}$ and subsequently fix the constant $c$ representing a bound for each of the simulated paths. Theoretically, we then work with $v$ since we need its boundedness to motivate the algorithm and in order to derive the error bound in Theorem 5.1. The new choice of $\bar{\sigma}$ of course leads to a larger $c$. According to the simulated paths, $c \geq 1.1$ is suitable. Picking $c=1.1$, we can see that the relative error in Table 6.4 is basically the same as in Table 6.1 such that a higher volatility parameter does not really affect the relative error. Moreover, choosing $c=2$ gives us an error that is only slightly worse such that we observe a relatively good robustness in $c$. Interestingly, we can also select $c=0.6$ as a parameter in the Gramians $P_{T}$ and $Q_{T}$ and run the model reduction procedure based on these nonadmissible Gramians and even get a small gain. It might be because the majority of the paths of $\bar{v}$ stay below the bound of $c=0.6$. Therefore, most of the dynamics are still captured in the reduced system. Although we have seen a relatively robust scheme in the parameter $c$, we suggest to not choose it too small or large in practice.

Table 6.4

Relative $L^{2}$-error for an enlarged volatility parameter $\bar{\sigma}=0.25$ with $\mathbf{K}=\mathbf{K}_{0}$ using $c=0.6,1.1,2$.

\begin{tabular}{|c|c|c|c|}
\hline & \multicolumn{3}{|c|}{$\|y-\tilde{y}\|_{L^{2}} /\|y\|_{L^{2}}$} \\
\hline$\tilde{n}$ & $c=0.6$ & $c=1.1$ & $c=2$ \\
\hline \hline 1 & $5.23 \mathrm{e}-03$ & $5.27 \mathrm{e}-03$ & $5.47 \mathrm{e}-03$ \\
4 & $2.06 \mathrm{e}-03$ & $2.09 \mathrm{e}-03$ & $2.22 \mathrm{e}-03$ \\
10 & $7.16 \mathrm{e}-04$ & $7.28 \mathrm{e}-04$ & $7.68 \mathrm{e}-04$ \\
\hline
\end{tabular}

6.2. Approximation error in the payoff. We have seen in section 6.1 that the quantity of interest $y$ of system (2.1), which we specified in (6.4), can be well approximated by the 
output $\tilde{y}$ of the reduced system (4.4) in a path-wise sense on some interval $[0, T]$. However, it is often of interest to consider weak errors instead. Therefore, we consider the following payoff function

$$
f(y)=\max \{y-K, 0\}
$$

that plays a role in the context of European call options, where $K$ denotes the strike price. We compare the expected payoff $\mathbb{E} f(y(T))$ at time $T$ with the one associated with the reduced system, which is $\mathbb{E} f(\tilde{y}(T))$, for $K=\left\langle\mathbf{1}, x_{0}\right\rangle_{2}$ in Figure 6.3. The relative errors in the expected payoff are larger if the correlations between the noise processes are small. The approximation works best if $\mathbf{K}=\mathbf{1 1}^{T}$. As displayed in Figure 6.3, the error is around 5e-06 for $\tilde{n}=2$ and our simulations also show an error of $5 \mathrm{e}-13$ already for $\tilde{n}=4$. If smaller correlations are involved, a larger $\tilde{n}$ needs to be chosen. However, selecting $4 \leq \tilde{n} \leq 10$ for $\mathbf{K}=\mathbf{K}_{0}$ already leads to a good estimate of the original payoff.

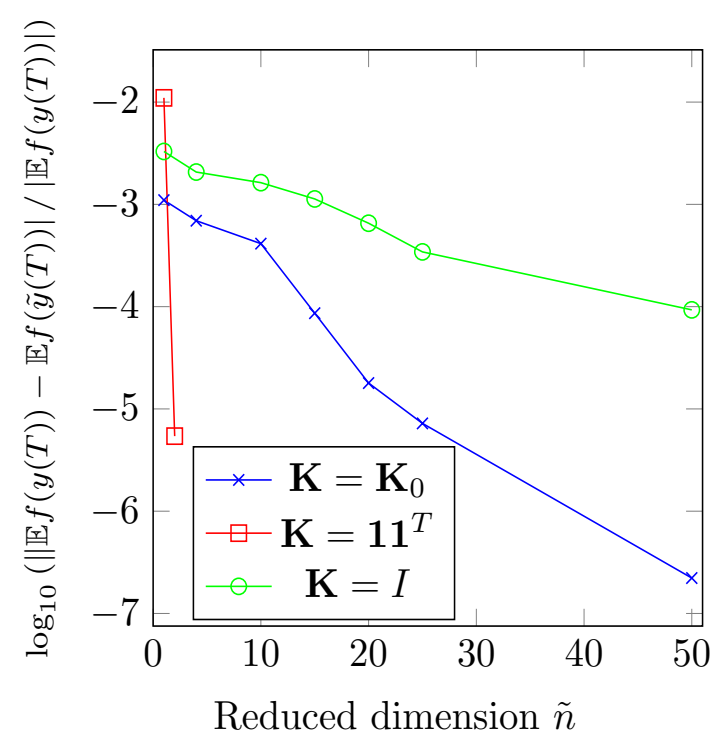

Figure 6.3. Logarithmic relative error expected payoff for $K=\left\langle\mathbf{1}, x_{0}\right\rangle_{2}$ and different reduced dimensions.

Looking at Table 6.5 we observe that weak error (error in the expected payoff) is of the same or of smaller error than the strong error in Table 6.1. Moreover, it can be seen that the approximation in the payoff is better if the strike price is below the value of the basket $\left\langle\mathbf{1}, x_{0}\right\rangle_{2}$ at time zero and it is worse if the strike price is above $\left\langle\mathbf{1}, x_{0}\right\rangle_{2}$.

So far, the weak error has not yet been analyzed concerning error bounds. We believe that it requires advanced techniques to succeed in this direction.

6.3. Applications to Bermudan options. We have seen a good path-wise performance of our method in section 6.1 and an even better approximation in the payoff in this section. Therefore, we see the potential of pricing high-dimensional Bermudan options with the help of MOR. Classical regression-based schemes as in $[32,43]$ cannot accurately determine values of Bermudan options with high-dimensional underlying asset models due to the curse of 
Table 6.5

Relative error in the payoff function for $\mathbf{K}=\mathbf{K}_{0}$ and different strike prices $K$.

\begin{tabular}{|c|c|c|c|}
\hline & \multicolumn{3}{|c|}{$|\mathbb{E} f(y(T))-\mathbb{E} f(\tilde{y}(T))| /|\mathbb{E} f(y(T))|$} \\
\hline$\tilde{n}$ & $K=0.9\left\langle\mathbf{1}, x_{0}\right\rangle_{2}$ & $K=\left\langle\mathbf{1}, x_{0}\right\rangle_{2}$ & $K=1.1\left\langle\mathbf{1}, x_{0}\right\rangle_{2}$ \\
\hline \hline 1 & $9.54 \mathrm{e}-04$ & $1.10 \mathrm{e}-03$ & $1.98 \mathrm{e}-02$ \\
4 & $8.81 \mathrm{e}-04$ & $6.92 \mathrm{e}-04$ & $5.28 \mathrm{e}-03$ \\
10 & $2.78 \mathrm{e}-04$ & $4.13 \mathrm{e}-04$ & $8.80 \mathrm{e}-04$ \\
15 & $3.41 \mathrm{e}-05$ & $8.63 \mathrm{e}-05$ & $2.22 \mathrm{e}-04$ \\
20 & $8.62 \mathrm{e}-06$ & $1.79 \mathrm{e}-05$ & $2.25 \mathrm{e}-05$ \\
25 & $2.56 \mathrm{e}-06$ & $7.22 \mathrm{e}-06$ & $2.19 \mathrm{e}-05$ \\
50 & $1.12 \mathrm{e}-07$ & $2.22 \mathrm{e}-07$ & $7.09 \mathrm{e}-07$ \\
\hline
\end{tabular}

dimensionality. However, reducing the asset price model in its dimension and subsequently applying the methods in $[32,43]$ can be promising. To fix notation, consider an optimal stopping problem with possible exercise date $\mathcal{J} \subset[0, T]$ with value

$$
u:=\sup _{\tau \in \mathcal{T}} \mathbb{E}[f(y(\tau))]
$$

where $\mathcal{T}$ denotes the set of all stopping times with respect to (w.r.t.) the filtration $\left(\mathcal{F}_{t}\right)_{t \in \mathcal{J}}$ generated by the Brownian motions $\bar{B}, W_{1}, \ldots, W_{q}$ restricted to $\mathcal{J}$. (Here, $\mathcal{J}$ is finite in the case of a Bermudan option, while $\mathcal{J}=[0, T]$ in the case of an American option. In all cases, we assume that $0 \in \mathcal{J}$.) Let us further consider the same problem in the reduced model,

$$
\tilde{u}:=\sup _{\tau \in \mathcal{T}} \mathbb{E}[f(\tilde{y}(\tau))] .
$$

Note that we choose the same class of stopping times for the full and for the reduced model, guaranteeing that the optimal stopping time for either choice of the model will be a suboptimal stopping time for the other choice of model.

Remark 7. In contrast to (6.5) and (6.6), one could also consider restricting the class of admissible stopping times to stopping times w.r.t. the filtration $\mathbb{G}:=\left(\mathcal{G}_{t}\right)_{t \in \mathcal{J}}$ generated by $v$ and $\tilde{x}$ for the reduced model and stopping times w.r.t. the filtration $\mathbb{H}:=\left(\mathcal{H}_{t}\right)_{t \in \mathcal{J}}$ generated by $v$ and $x$ for the full model, respectively. In general, this means that optimal stopping times for either model will not even be candidate stopping times for the other model. Often - but not always - $\mathbb{H}$ will be an enlargement of $\mathbb{G}$, and under some conditions, this would allow us to understand stopping times for the full model as randomized stopping times for the reduced model in the sense of [21]. This implies that the optimal stopping time for the full model is a suboptimal randomized stopping time for the reduced model, and its expected payoff is less or equal to the expected payoff of the optimal stopping time of the reduced model w.r.t. the smaller filtration. In general, however, the analysis of the behavior of American options for general model reductions within their own generated filtrations is beyond the scope of this paper.

Lemma 6.1. Assume that the reduced model is close to the full model in the sense that

$$
\mathbb{E}\left[\sup _{t \in \mathcal{J}}|f(y(t))-f(\tilde{y}(t))|\right] \leq \epsilon .
$$

Copyright $\odot$ by SIAM. Unauthorized reproduction of this article is prohibited. 
We then have that

$$
|u-\tilde{u}| \leq \epsilon
$$

Proof. Let $\tau^{*}$ denote the optimal stopping time for the full problem, i.e., $u=\mathbb{E}\left[f\left(y\left(\tau^{*}\right)\right)\right]$. Similarly, let $\tilde{\tau}^{*}$ denote the optimal stopping time for the reduced problem. We have

$$
\begin{aligned}
u-\tilde{u} & =\mathbb{E}\left[f\left(y\left(\tau^{*}\right)\right)\right]-\mathbb{E}\left[f\left(\tilde{y}\left(\tilde{\tau}^{*}\right)\right)\right] \\
& =\underbrace{\mathbb{E}\left[f\left(y\left(\tau^{*}\right)\right)\right]-\mathbb{E}\left[f\left(y\left(\tilde{\tau}^{*}\right)\right)\right]}_{\geq 0}+\underbrace{\mathbb{E}\left[f\left(y\left(\tilde{\tau}^{*}\right)\right)\right]-\mathbb{E}\left[f\left(\tilde{y}\left(\tilde{\tau}^{*}\right)\right)\right]}_{\geq-\epsilon} \\
& \geq-\epsilon,
\end{aligned}
$$

since $\tilde{\tau}^{*}$ is a suboptimal stopping time for $f(y)$.

For the upper bound, we note that $\tau^{*}$ is a suboptimal stopping time for $f(\tilde{y})$ and obtain

$$
\begin{aligned}
u-\tilde{u} & =\mathbb{E}\left[f\left(y\left(\tau^{*}\right)\right)\right]-\mathbb{E}\left[f\left(\tilde{y}\left(\tilde{\tau}^{*}\right)\right)\right] \\
& =\underbrace{\mathbb{E}\left[f\left(y\left(\tau^{*}\right)\right)\right]-\mathbb{E}\left[f\left(\tilde{y}\left(\tau^{*}\right)\right)\right]}_{\leq \epsilon}+\underbrace{\mathbb{E}\left[f\left(\tilde{y}\left(\tau^{*}\right)\right)\right]-\mathbb{E}\left[f\left(\tilde{y}\left(\tilde{\tau}^{*}\right)\right)\right]}_{\leq 0} \\
& \leq \epsilon,
\end{aligned}
$$

since $\tilde{\tau}^{*}$ is optimal for $f(\tilde{y})$.

Remark 8. Unfortunately, the $L^{2}$-error bound obtained in Theorem 5.1 is not a candidate for $\epsilon$ in (6.7) since we cannot bound the $L^{\infty}$-error by the $L^{2}$-error. However, we can usually estimate $\mathbb{E}\left[\sup _{t \in \mathcal{J}}|f(y(t))-f(\tilde{y}(t))|\right]$ empirically by sampling $y$ and $\tilde{y}$. Indeed, for numerical purposes, we may always assume that $\mathcal{J}$ is finite, such that the $L^{\infty}$-norm for a given trajectory is easily computable. Additionally, we note that estimating $\mathbb{E}\left[\sup _{t \in \mathfrak{J}}|f(y(t))-f(\tilde{y}(t))|\right]$ is, of course, much easier than estimating $|u-\tilde{u}|$, as no optimal stopping problem needs to be solved. This type of approximation for the bound between the option prices $u$ and $\tilde{u}$ is also used in the numerical example below. Theoretical error bounds of the form (6.7) would require very different techniques than applied in the proof of Theorem 5.1 and are left for further research.

We now create an example, in which the error in (6.7) is already relatively low for a reduced dimension $\tilde{n}=5$, since this gives us the certainty that the value of the Bermudan option $\tilde{u}$ in the reduced model is close to the actual value $u$ by Lemma 6.1. To do so, we modify (6.1) by choosing $n=30$, a constant volatility and dividends resulting in the following Black-Scholes model:

$$
d x_{i}(t)=(\mathrm{r}-\delta) x_{i}(t) d t+\xi x_{i}(t) d W_{i}(t), \quad x_{i}(0)=x_{0, i}, \quad t \in[0, T],
$$

where $\mathrm{r}=0.02, \delta=0.07, \xi=0.2, T=1$, and $i=1, \ldots, n$. The noise processes are correlated. Their covariance matrix is generated the same way as $\mathbf{K}_{0}$ above, and we choose randomly generated initial values $x_{0, i} \in[0.15,2.5]$. The quantity of interest is chosen as before; i.e.,

$$
y(t)=\sum_{i=1}^{n} x_{i}(t),
$$

Copyright $\odot$ by SIAM. Unauthorized reproduction of this article is prohibited. 
and the following discounted payoff function is considered

$$
f(y(t))=\mathrm{e}^{-\mathrm{r} t} \max \{y(t)-K, 0\}
$$

with $K=\left\langle\mathbf{1}, x_{0}\right\rangle_{2}$. We have five exercise dates of the associated Bermudan option, i.e., $\mathcal{J}=\{0,0.25,0.5,0.75,1\}$.

We apply the MOR technique described in section 4 in order to obtain the reduced system (4.4) with dimensions $\tilde{n}=1, \ldots, 5$, Subsequently, we determine the error in (6.7). These errors are stated in the third column in Table 6.6 showing that we are already very close to the actual value $u$ using a reduced system with dimension $\tilde{n}=5$. In order to determine the fair price for the Bermudan option in the reduced model $(\tilde{n}=1, \ldots, 5)$, we apply the algorithm of Longstaff and Schwartz [32]. Within this regression approach Hermite polynomials of absolute order up to 5 are used as a basis. Moreover, the payoff function is included in the basis as well. This leads to the values $\tilde{u}$ in the second column of Table 6.6. The gain in $\tilde{u}$ from $\tilde{n}=1$ to $\tilde{n}=5$ is relatively low. It is hard to distinguish between both values knowing that the standard deviation of this estimation is 0.00105 which is the same order as the gain. Observing the values $\tilde{u}$ in Table 6.6 for different reduced order dimensions, it seems that the corresponding bound is not very tight such that we suppose that the deviation between $u$ and $\tilde{u}$ is much lower than $10^{-2}$ when choosing $\tilde{n}=5$. Moreover, notice that the value of the European option in the above asset price model is 0.87980 , such that there is a significant difference between both option prices. This makes our method beneficial since computing the Bermudan option price in the reduced model leads to a large gain in comparison to the European option price in the full model which would be a good estimator if the values of both types of options do not deviate too much.

Table 6.6

Value Bermudan option based on the reduced asset price model corresponding to (6.8) and associated error bounds from Lemma 6.1 .

\begin{tabular}{|c|c|c|}
\hline$\tilde{n}$ & Value $\tilde{u}$ of Bermudan option reduced system & $\mathbb{E}\left[\sup _{t \in \mathcal{J}}|f(y(t))-f(\tilde{y}(t))|\right]$ \\
\hline \hline 1 & 0.99173 & 0.079619 \\
2 & 0.99260 & 0.049844 \\
3 & 0.99313 & 0.025734 \\
4 & 0.99295 & 0.016036 \\
5 & 0.99354 & 0.012181 \\
\hline
\end{tabular}

7. Conclusions and outlook. In this paper, we have shown that MOR can be an effective technique to construct lower-dimensional surrogate models of large-scale financial models. These surrogate models can be tackled by higher-order computational methods than Monte Carlo simulation. We construct a specific path-wise MOR method and test it in a multidimensional Heston model. The MOR turns out to work very well for European basket option pricing, especially when the individual assets are strongly correlated (a very realistic scenario). For instance, in a common Doust-type correlation regime, for $n=100$ assets, a reduced model with dimension $\tilde{n}=1$ was able to capture the price of an ITM basket option up to a relative error of $10^{-3}$, whereas for an OTM option we obtained the same error bound with $\tilde{n}=10$, which is still a very significant dimension reduction. 
Of course, this paper only scratches the surface of applications of MOR in finance. In particular, we identify two very relevant extensions that will be highly beneficial in a financial context. On the one hand, consider that we have restricted ourselves to linear dynamics and essentially linear payoff functions - in the sense that the payoff is assumed to be a nonlinear function of a low-dimensional projection of the full price process. Both restrictions can be quite relevant in finance. Allowing nonlinear dynamics opens up the possibility of including the stochastic variance process in the model order reduction, as well as having local volatility components. Techniques for MOR in nonlinear dynamics have already been developed in the deterministic case $[10,11,29]$ and have been extended to stochastic differential equations in some special cases [34]. General nonlinear payoff functions are also relevant in finance, think of max-call options. One strategy already available in our framework is to choose $C$ to be the identity matrix.

On the other hand, note that the MOR framework developed in this paper is strong in the probabilistic sense; i.e., we try to approximate the process itself. In many financial application, we are interested in weak approximations; i.e., we want to approximate the distribution of the process. As this is a much weaker concept, even better MOR techniques are conceivable. However, developing an appropriate framework does not seem obvious, and it is unclear how to proceed in this direction.

Appendix A. Resolvent positive operators. Let $\left(H^{n},\langle\cdot, \cdot\rangle_{F}\right)$ be the Hilbert space of symmetric $n \times n$ matrices, where $\left\langle M_{1}, M_{2}\right\rangle_{F}:=\operatorname{tr}\left(M_{1}^{T} M_{2}\right)$ is the Frobenius inner product of two matrices $M_{1}$ and $M_{2}$. The corresponding norm is defined by $\left\|M_{1}\right\|_{F}^{2}:=\left\langle M_{1}, M_{1}\right\rangle_{F}$. Moreover, let $H_{+}^{n}$ be the subset of symmetric positive semidefinite matrices. We now define positive and resolvent positive operators on $H^{n}$.

Definition A.1. A linear operator $L: H^{n} \rightarrow H^{n}$ is called positive if $L\left(H_{+}^{n}\right) \subset H_{+}^{n}$. It is resolvent positive if there is an $\alpha_{0} \in \mathbb{R}$ such that for all $\alpha>\alpha_{0}$ the operator $(\alpha I-L)^{-1}$ is positive.

The operator $\mathcal{L}(X):=A X+X A^{T}$ is resolvent positive for $A \in \mathbb{R}^{n \times n}$ which is, e.g., shown in [17]. Moreover, $\Pi(X):=c \sum_{i, j=1}^{q} N_{i} X N_{j}^{T} k_{i j}$ is positive for $N_{i} \in \mathbb{R}^{n \times n}$ by [35, Proposition 5.3]. This implies that the generalized Lyapunov operator $\mathcal{L}+\Pi$ is resolvent positive. We now state an equivalent characterization for resolvent positive operators in the following. It can be found in a more general form in [17, 20,39].

Theorem A.2. A linear operator $L: H^{n} \rightarrow H^{n}$ is resolvent positive if and only if $\left\langle V_{1}, V_{2}\right\rangle_{F}=$ 0 implies $\left\langle L V_{1}, V_{2}\right\rangle_{F} \geq 0$ for $V_{1}, V_{2} \in H_{+}^{n}$.

Appendix B. Pending proofs. We prove Lemmas 2.1 and 2.3 in the following two subsections.

B.1. Proof of Lemma 2.1. We apply Ito's product rule to $x(t) x^{T}(t)$ and obtain

$$
d\left(x(t) x^{T}(t)\right)=d x(t) x^{T}(t)+x(t) d x^{T}(t)+d x(t) d x^{T}(t) .
$$

Inserting (2.1a) yields

Copyright (c) by SIAM. Unauthorized reproduction of this article is prohibited. 


$$
\begin{aligned}
d x(t) x^{T}(t)+x(t) d x^{T}(t)= & A x(t) x^{T}(t) d t+\sum_{i=1}^{q} \sqrt{v(t)} N_{i} x(t) x^{T}(t) d W_{i}(t) \\
& +x(t) x^{T}(t) A^{T} d t+\sum_{i=1}^{q} \sqrt{v(t)} x(t) x^{T}(t) N_{i}^{T} d W_{i}(t)
\end{aligned}
$$

With (2.1a) and using that $d W_{i}(t) d W_{j}(t)=k_{i j} d t$, we find

$$
d x(t) d x^{T}(t)=v(t) \sum_{i, j=1}^{q} N_{i} x(t) x^{T}(t) N_{j}^{T} k_{i j} d t
$$

Let $e_{i}$ denote the $i$ th unit vector. Then, we have

$$
k_{i j}=e_{i}^{T} \mathbf{K}^{\frac{1}{2}} \mathbf{K}^{\frac{1}{2}} e_{j}=\sum_{k=1}^{q}\left\langle\mathbf{K}^{\frac{1}{2}} e_{i}, e_{k}\right\rangle_{2}\left\langle\mathbf{K}^{\frac{1}{2}} e_{j}, e_{k}\right\rangle_{2} .
$$

Using this fact, we obtain that

$$
\sum_{i, j=1}^{q} N_{i} x(t) x^{T}(t) N_{j}^{T} k_{i j}=\sum_{k=1}^{q}\left(\sum_{i=1}^{q} N_{i} x(t)\left\langle\mathbf{K}^{\frac{1}{2}} e_{i}, e_{k}\right\rangle_{2}\right)\left(\sum_{j=1}^{q} N_{j} x(t)\left\langle\mathbf{K}^{\frac{1}{2}} e_{j}, e_{k}\right\rangle_{2}\right)^{T} \geq 0
$$

is a positive semidefinite matrix. Hence, we can enlarge the right side of (B.2) by replacing $v$ by its bound $c$. This leads to

$$
d x(t) d x^{T}(t) \leq c \sum_{i, j=1}^{q} N_{i} x(t) x^{T}(t) N_{j}^{T} k_{i j} d t
$$

We apply the expected value to both sides of (B.1) and (B.3). Since the Ito integrals have mean zero, we have

$$
\frac{d}{d t} \mathbb{E}\left[x(t) x^{T}(t)\right] \leq A \mathbb{E}\left[x(t) x^{T}(t)\right]+\mathbb{E}\left[x(t) x^{T}(t)\right] A^{T}+c \sum_{i, j=1}^{q} N_{i} \mathbb{E}\left[x(t) x^{T}(t)\right] N_{j}^{T} k_{i j},
$$

which concludes the proof.

B.2. Proof of Lemma 2.3. We combine (2.4) with (2.5) and obtain

$$
\dot{Y}(t) \geq L(Y(t))
$$

where $Y:=Z-X$. We define the difference function $D(t):=\dot{Y}(t)-L(Y(t)) \geq 0$ and consider the following perturbed differential equation

$$
\dot{Y}_{\epsilon}(t)=L\left(Y_{\epsilon}(t)\right)+D(t)+\epsilon I
$$

with parameter $\epsilon \geq 0$ and initial state $Y_{\epsilon}(0)=Y(0)+\epsilon I$. We see that $Y_{0}(t)=Y(t)$ for all $t \in[0, T]$ since $Y_{0}-Y$ solves (2.5) with initial condition zero. Since $Y_{\epsilon}$ continuously depends on $\epsilon$ and the initial data, we have $\lim _{\epsilon \rightarrow 0} Y_{\epsilon}(t)=Y_{0}(t)=Y(t)$ for all $t \in[0, T]$. 
We want to prove that $Y_{\epsilon}(t)$ is positive definite for all $t$ and all $\epsilon>0$. To do so, let us assume the converse, i.e., there is a $\tilde{u} \neq 0$ and a $\tilde{t}>0$ such that $\tilde{u}^{T} Y_{\epsilon}(\tilde{t}) \tilde{u} \leq 0$. We know that $f_{\epsilon}(u, t):=u^{T} Y_{\epsilon}(t) u$ is positive at $t=0$ for all $u \in \mathbb{R}^{n} \backslash\{0\}$ since $Y(0) \geq 0$ by assumption. Since $f_{\epsilon}$ is nonpositive in some point $(\tilde{u}, \tilde{t})$ and due to the continuity of $t \mapsto Y_{\epsilon}(t)$, there is a point $t_{0} \in(0, \tilde{t}]$ for which

$$
u_{0}^{T} Y_{\epsilon}\left(t_{0}\right) u_{0}=0 \quad \text { and } \quad u_{0}^{T} Y_{\epsilon}(t) u_{0}>0, \quad t<t_{0}
$$

for some $u_{0} \neq 0$, whereas $u^{T} Y_{\epsilon}\left(t_{0}\right) u \geq 0$ for all other $u \in \mathbb{R}^{n}$. Since $L$ is resolvent positive, $0=$ $u_{0}^{T} Y_{\epsilon}\left(t_{0}\right) u_{0}=\left\langle Y_{\epsilon}\left(t_{0}\right), u_{0} u_{0}^{T}\right\rangle_{F}$ implies $0 \leq\left\langle L\left(Y_{\epsilon}\left(t_{0}\right)\right), u_{0} u_{0}^{T}\right\rangle_{F}=u_{0}^{T} L\left(Y_{\epsilon}\left(t_{0}\right)\right) u_{0}$ by Theorem A.2. Hence, we have

$$
\left.u_{0}^{T} \dot{Y}_{\epsilon}(t) u_{0}\right|_{t=t_{0}}=u_{0}^{T} L\left(Y_{\epsilon}\left(t_{0}\right)\right) u_{0}+u_{0}^{T} D\left(t_{0}\right) u_{0}+\epsilon\left\|u_{0}\right\|_{2}^{2}>0 .
$$

Consequently, we know that there are $t<t_{0}$ close to $t_{0}$ for which $u_{0}^{T} Y_{\epsilon}(t) u_{0}<0$. This contradicts (B.4) and hence our assumption is wrong such that $Y_{\epsilon}(t)$ is positive definite for all $t \in[0, T]$ and $\epsilon>0$. Taking the limit of $\epsilon \rightarrow 0$, we obtain $Y(t) \geq 0$ for all $t \in[0, T]$ which concludes the proof.

Acknowledgments. The authors would like to thank the anonymous reviewers for their helpful, constructive, and detailed comments that greatly contributed to improving the paper.

\section{REFERENCES}

[1] A. C. Antoulas, Approximation of Large-Scale Dynamical Systems, Adv. Des. Control 6, SIAM Publications, Philadelphia, PA, 2005.

[2] A. C. Antoulas, C. A. Beattie, and S. Gugercin, Interpolatory Methods for Model Reduction, SIAM, Philadelphia, 2020.

[3] R. H. Bartels and G. W. Stewart, Solution of the matrix equation $A X+X B=C$, Comm. ACM, 15 (1972), pp. 820-826.

[4] C. BAyer, J. Häppölä, And R. Tempone, Implied stopping rules for American basket options from Markovian projection, Quant. Finance, 19 (2019), pp. 371-390.

[5] C. Bayer, M. Siebenmorgen, And R. Tempone, Smoothing the payoff for efficient computation of basket option prices, Quant. Finance, 18 (2018), pp. 491-505.

[6] C. A. Beattie, S. Gugercin, and V. Mehrmann, Model reduction for systems with inhomogeneous initial conditions, Syst. Control Lett., 99 (2017), pp. 99-106.

[7] S. Becker, C. Hartmann, M. Redmann, and L. Richter, Feedback Control Theory Ej Model Order Reduction for Stochastic Equations, preprint, arXiv:1912.06113, 2019.

[8] P. Benner And T. Breiten, Low rank methods for a class of generalized Lyapunov equations and related issues, Numer. Math., 124 (2013), pp. 441-470.

[9] P. Benner And T. DAmm, Lyapunov equations, energy functionals, and model order reduction of bilinear and stochastic systems, SIAM J. Control Optim., 49 (2011), pp. 686-711.

[10] P. Benner and P. Goyal, Balanced Truncation Model Order Reduction for Quadratic-Bilinear Control Systems, preprint, arXiv:1705.00160, 2017.

[11] P. Benner And P. Goyal, Interpolation-Based Model Order Reduction for Polynomial Parametric Systems, preprint, arXiv:1904.11891, 2019.

[12] P. Benner, M. Ohlberger, A. Cohen, And K. Willcox, eds., Model Reduction and Approximation: Theory and Algorithms, SIAM, Philadelphia, PA, 2017.

[13] P. Benner and M. Redmann, Model reduction for stochastic systems, Stoch PDE: Anal Comp, 3 (2015), pp. 291-338.

[14] G. Brunick And S. Shreve, Mimicking an Itô process by a solution of a stochastic differential equation, Ann. Appl. Probab., 23 (2013), pp. 1584-1628.

Copyright (c) by SIAM. Unauthorized reproduction of this article is prohibited. 
[15] H. Buehler, L. Gonon, J. Teichmann, and B. Wood, Deep hedging, Quant. Finance, 19 (2019), pp. 1271-1291.

[16] H. Bungartz and M. Griebel, Sparse grids, Acta Numerica, 13 (2004), pp. 147-269.

[17] T. DAmm, Rational Matrix Equations in Stochastic Control, Lecture Notes in Control and Inform. Sci. 297, Springer, Berlin, 2004.

[18] T. DAmm, Direct methods and ADI-preconditioned Krylov subspace methods for generalized Lyapunov equations, Numer. Linear Algebra Appl., 15 (2008), pp. 853-871.

[19] P. Doust, Modelling discrete probabilities, Quantitative Anal. Group, RBS, 2007.

[20] L. ElSNeR, Quasimonotonie und Ungleichungen in halbgeordneten Räumen, Linear Algebra Appl., 8 (1974), pp. 249-261.

[21] I. GYÖNGY AND D. ŠIšKA, On randomized stopping, Bernoulli, 14 (2008), pp. 352-361.

[22] W. Gawronski And J. JuAng, Model reduction in limited time and frequency intervals, Int. J. Syst. Sci., 21 (1990), pp. 349-376.

[23] T. Gerstner, Sparse grid quadrature methods for computational finance, Habilitation, University of Bonn, 2007.

[24] S. Gugercin And A. C. Antoulas, A survey of model reduction by balanced truncation and some new results, Internat. J. Control, 77 (2004), pp. 748-766.

[25] I. GYÖNGY, Mimicking the one-dimensional marginal distributions of processes having an Itô differential, Probab. Theory Related Fields, 71 (1986), pp. 501-516.

[26] B. Hambly, M. Mariapragassam, and C. Reisinger, A forward equation for barrier options under the Brunick ES Shreve Markovian projection, Quant. Finance, 16 (2016), pp. 827-838.

[27] J. Han, A. Jentzen, And E. Weinan, Solving high-dimensional partial differential equations using deep learning, Proc. Nat. Acad. Sci., 115 (2018), pp. 8505-8510.

[28] R. Z. Khasminskir, Stochastic stability of differential equations, Monographs and Textbooks on Mechanics of Solids and Fluids. Mechanics: Analysis, 7, Sijthoff \& Noordhoff, Alphen aan den Rijn, The Netherlands; Rockville, Maryland, USA, 1980.

[29] B. Krämer AND K. Willcox, Balanced Truncation Model Reduction for Lifted Nonlinear Systems, preprint, arXiv:1907.12084, 2019.

[30] D. KResSner And P. Sirković, Truncated low-rank methods for solving general linear matrix equations, Numer. Linear Algebra Appl., 22 (2015), pp. 564-583.

[31] P. Kürschner, Balanced truncation model order reduction in limited time intervals for large systems, Adv. Comput. Math., (2018), pp. 1-24.

[32] F. A. Longstaff And E. S. Schwartz, Valuing American options by simulation: A simple least-squares approach, Rev. Financial Studies, 14 (2001), pp. 113-147.

[33] B. C. Moore, Principal component analysis in linear systems: Controllability, observability, and model reduction, IEEE Trans. Autom. Control, AC-26 (1981), pp. 17-32.

[34] M. Redmann, Energy estimates and model order reduction for stochastic bilinear systems, Internat. J. Control, 93 (2020), 1954-1963.

[35] M. Redmann, Type II singular perturbation approximation for linear systems with Lévy noise, SIAM J. Control Optim., 56 (2018), pp. 2120-2158.

[36] M. Redmann, Bilinear Systems - A New Link to $\mathcal{H}_{2}$-Norms, Relations to Stochastic Systems and Further Properties, preprint, arXiv:1910.14427, 2020.

[37] M. Redmann, An $L_{T}^{2}$-error bound for time-limited balanced truncation, Syst. Control Lett., 136 (2020), 104620.

[38] M. Redmann And P. Kürschner, An output error bound for time-limited balanced truncation, Syst. Control Lett., 121 (2018), pp. 1-6.

[39] H. Schneider and M. Vidyasagar, Cross-Positive Matrices, SIAM J. Numer. Anal., 7 (1970), pp. 508519.

[40] I. SchuR, Bemerkungen zur Theorie der beschränkten Bilinearformen mit unendlich vielen Veränderlichen, Journal für die reine und angewandte Mathematik, 140 (1911), pp. 1-28.

[41] S. D. Shank, V. Simoncini, And D. B. Szyld, Efficient low-rank solution of generalized Lyapunov equations, Numer. Math., 134 (2016), pp. 327-342.

[42] V. Simoncini, Computational methods for linear matrix equations, SIAM Rev., 58 (2016), pp. 377-441.

[43] J. Tsitsiklis And B. VAn Roy, Regression methods for pricing complex American style options, IEEE Trans. Neural. Net., 12 (2001), pp. 694-703.

Copyright (c) by SIAM. Unauthorized reproduction of this article is prohibited. 